\title{
ASYMPTOTIC BEHAVIOR AND TRAVELING WAVE SOLUTIONS FOR PARABOLIC FUNCTIONAL DIFFERENTIAL EQUATIONS
}

\author{
KLAUS W. SCHAAF
}

\begin{abstract}
This paper is a generalization of the theory of the KPP and bistable nonlinear diffusion equations. It is shown that traveling wave solutions exist for nonlinear parabolic functional differential equations (FDEs) which behave very much like the well-known solutions of the classical KPP and bistable equations. Among the techniques used are maximum principles, sub- and supersolutions, phase plane techniques for FDEs and perturbation of linear operators.
\end{abstract}

1. Preliminaries. Since the classical paper by Kolmogorov et al. [24] there have been intensive studies on the asymptotic behavior of solutions to the Cauchy problem

$$
\begin{aligned}
& \partial_{t} u-\partial_{x}^{2} u=f(u), \quad x \in \mathbf{R}, t>0, \\
& 0 \leq u(x, 0)=u_{0}(x) \leq 1, \quad x \in \mathbf{R},
\end{aligned}
$$

with the general condition on $f$

$$
f(0)=f(1)=0, \quad f \in C^{2}([0,1], \mathbf{R}) .
$$

Depending on the shape of $f$, the solutions of (1.1-3) show characteristic asymptotic behavior (see e.g. [10] for more details).

$1.1 \mathrm{KPP}$-equation. For $f$ positive and convex on $] 0,1[$ there is a critical positive value $c$ depending only on $f^{\prime}(0)>0$ with the following properties:

(i) $c^{*}$ is minimal velocity [10], i.e. for all $c \geq c^{*}$ there are nontrivial traveling wave solutions

$$
u(x, t)=\varphi_{c}(x+c t)
$$

with

$$
0<\varphi_{c}<1, \quad \lim _{s \rightarrow \infty} \varphi_{c}(s)=1, \quad \lim _{s \rightarrow-\infty} \varphi_{c}(s)=0 ;
$$

for $c<c^{*}$, there are only the trivial wave solutions $\varphi \equiv 0$ and $\varphi \equiv 1$.

(ii) $c^{*}$ is asymptotic speed of propagation [24], i.e. for a solution of $(1.1-3)$ with compact support of $u_{0}$ we have

$$
\lim _{t \rightarrow \infty} u(x \pm c t, t)= \begin{cases}1 & \text { for } 0 \leq|c|<c^{*} \\ 0 & \text { for }|c|>c^{*}\end{cases}
$$

(iii) $c^{*}$ can be calculated as

$$
c^{*}=\inf \left\{c>0 \mid \Delta_{c} \text { has a real zero }\right\}
$$

Received by the editors January 23, 1986 and, in revised form, June 3, 1986.

1980 Mathematics Subject Classification (1985 Revision). Primary 35K10, 35K55, 34K 10. 
where

$$
\Delta_{c}(\lambda)=\lambda^{2}-c \lambda+f^{\prime}(0)
$$

is the characteristic equation of the linearization of the "traveling wave equation"

$$
\varphi_{c}^{\prime \prime}-c \varphi_{c}^{\prime}+f\left(\varphi_{c}\right)=0
$$

around zero.

(iv) The traveling waves of 1.1.1 are highly unstable and are stable only in spaces with appropriate weighted norms.

More information on this type of equation can be found e.g. in $[\mathbf{2 - 3}, \mathbf{5}, \mathbf{1 5}$, 19, 27, 34]. The situation looks somewhat different if $f$ changes sign (see e.g. [7, 11-12, 20-21].

1.2 Huxley equation. If $f$ admits an intermediate steady state $0<a<1$ such that

$$
f(a)=0, \quad f(s) \begin{cases}<0 & \text { for } 0<s<a, \\ >0 & \text { for } a<s<1,\end{cases}
$$

then there is a unique $c \in \mathbf{R}$ such that there exists a nontrivial traveling wave $\varphi=\varphi_{c}$ with the asymptotic behavior (1.5). $\varphi$ is stable with respect to perturbations in $B C^{0}(\mathbf{R}) \cap\{0 \leq u \leq 1\}$.

It turned out that a wide class of equations exhibit asymptotic behavior like the KPP equation described in $1.1-$ e.g. equations modeling epidemics or population dynamics, integral, integrodifferential or functional differential equations, e.g. [1, 8-9, 23, 30-31, 33, 35]. The simplest equation (next to (1.1)) is the differencedifferential equation

$$
\partial_{t} u-\partial_{x}^{2} u=f(u(x, t), u(x, t-\tau)), \quad \tau \in \mathbf{R}^{+},
$$

where $f$ satisfies the conditions

$$
f(0,0)=f(1,1)=0,
$$

thus allowing the steady state solution $u \equiv 0$ and $u \equiv 1$ of (1.11). Equation (1.11) with $f(r, s)=s(1-r)$ was derived in [23] from a branching process; in this model, $f$ fulfills the additional properties

$$
\begin{array}{rll}
\partial_{2} f(r, s) \geq 0 & \text { for } 0 \leq r, s \leq 1 & \text { (quasimonotonicity) } \\
f(r, s)>0 & \text { for } 0<r, s<1 & \text { (positivity). }
\end{array}
$$

Difference-differential equations of type $(1.11-13)$ are the main subject of this paper. In $\S 2$ we will show the existence of a minimal and asymptotic velocity in the sense of the KPP equation (1.1) and derive an instability criterion for the traveling waves.

This part uses the theory of functional differential equations [16] and maximum principles for parabolic functional differential equations [26, 32] and can therefore be done for more general functionals; for the sake of simplicity I restrict myself to equations (1.11) with one fixed time lag.

$\S 3$ treats the case where $f(r, s)$ allows an intermediate equilibrium $a$ between 0 and 1 . In this case we shall prove the existence of a unique (up to translation) traveling front with a unique velocity $c$; this velocity is determined by the global behavior of the nonlinearity $f$ and cannot be calculated explicitly. 
$\S 4$ investigates the position of the spectrum of the linearization of (1.11) around the traveling wave $\varphi_{c}$ (in an appropriate coordinate system) and represents a first step in the investigation of the stability of the traveling waves; the results of this part do not depend on the quasimonotonicity of the nonlinearity $f$.

This paper contains the main results of the author's Ph.D. thesis "Asymptotisches Verhalten und Wellenlösungen von semilinearen parabolischen Funktionaldifferentialgleichungen", Heidelberg, 1983; I would like to thank Professor Willi Jäger and my Heidelberg friends and colleagues for their helpful discussions, encouragement and friendship.

2. Positive nonlinearities. In this paper, we discuss the Cauchy problem

$$
\begin{aligned}
\partial_{t} w-\partial_{x}^{2} w & =f(w(x, t), w(x, t-\tau)), \quad x \in \mathbf{R}, t>0, \\
0 & \leq w(x, t) \leq 1, \quad x \in \mathbf{R}, t \leq 0,
\end{aligned}
$$

where $\tau$ is a fixed nonnegative number and $f$ satisfies the following

2.1 Hypotheses.

(i) $f \in C^{1, \nu}\left(\mathbf{R}^{2}, \mathbf{R}\right), f(0,0)=f(1,1)=0$.

(ii) $\partial_{2} f(r, s) \geq 0$ for $0 \leq r, s \leq 1$ (quasimonotonicity).

(iii) $f(r, s) \geq 0$ for $0 \leq r, s \leq 1$ and $f(r, r)>0$ for $0<r<1$ (positivity).

(iv) $\partial_{1} f(0,0)+\partial_{2} f(0,0)>0$.

One of the most important tools in this paragraph is the following

2.2 PROPOSITION (MAXIMUM PRINCIPLE). Let $f: \mathbf{R}^{2} \rightarrow \mathbf{R}$ be quasimonotone (see 2.1(ii)) and uniformly Lipschitz continuous, $\xi: \mathbf{R}^{2} \rightarrow \mathbf{R}$ continuous. Then the following holds: If $u, v$ are bounded regular solutions (i.e. all necessary derivatives are defined for $t>0$ ) of

$$
\begin{aligned}
& \partial_{t} u+c \partial_{x} u-\partial_{x}^{2} u \leq f(u(x, t), u(\xi(x, t), t-\tau)) \quad \text { for } x \in \mathbf{R}, t>0, \\
& \partial_{t} v+c \partial_{x} v-\partial_{x} v \geq f(v(x, t), v(\xi(x, t), t-\tau))
\end{aligned}
$$

with initial values

$$
u \leq v \quad \text { on } \mathbf{R} \times \mathbf{R}_{0}^{-}
$$

then

$$
u \leq v \quad \text { on } \mathbf{R} \times \mathbf{R}^{+}
$$

If there is $(x, t)$ with $t>0$ and $u(x, t)=v(x, t)$, then $u$ and $v$ are identical (strong maximum principle).

The maximum principle holds under much weaker conditions $[\mathbf{2 6}, \mathbf{3 2}]$; the decisive conditions are quasimonotonicity, (unilateral) Lipschitz conditions for $f$ and the fact that the functional $f$ does not depend on the "future". As an immediate consequence of the maximum principle we find that every nontrivial solution $u$ of (2.1-2) under the hypotheses 2.1 will satisfy

$$
0<u<1 \text { on } \mathbf{R} \times \mathbf{R}^{+} .
$$

To study the propagation properties of solutions $w$ to (2.1-2) we analyze the behavior of $w(x \pm c t, t), c \in \mathbf{R}$, for $t \rightarrow+\infty$, and because of the symmetry of $(2.1)$ 
we can restrict ourselves to $s=x+c t$ with $c \geq 0$. If $w$ solves $(2.1-2)$, then the function $W(s, t):=w(s-c t, t)$ solves the transformed equation

$$
\begin{gathered}
\partial_{t} W+c \partial_{s} W-\partial_{s}^{2} W=f(W(s, t), W(s-c \tau, t-\tau)), \quad s \in \mathbf{R}, t>0 \\
W(s, t)=w(s-c t, t), \quad t \leq 0
\end{gathered}
$$

Stationary solutions to (2.7) play an important role in the analysis of the asymptotic behavior of solutions to (2.1-2). We say

2.3 DEFINITION. A function $u \in C^{2}(\mathbf{R}, \mathbf{R})$ is called a wave solution (or traveling wave) with velocity $c$ iff

$$
u^{\prime \prime}(s)-c u^{\prime}(s)+f(u(s), u(s-c \tau)) \quad \text { for all } s \in \mathbf{R}
$$

and additionally

$$
0 \leq u \leq 1 \text { on } \mathbf{R} \text {. }
$$

The constants 0 and 1 are called trivial wave solutions.

To be able to apply Hale's theory [16] of functional differential equations (FDEs), we define for fixed $\tau \geq 0$

$$
f_{c}\left(u_{s}\right):=f(u(s), u(s-c \tau))
$$

as a function $f_{c}: C_{1}:=C^{0}\left([-T, 0], \mathbf{R}^{1}\right) \rightarrow \mathbf{R} . T$ is a positive fixed number greater or equal to $c \tau$, and unless otherwise stated we will be able to take $T=c \tau$ so that we may write $C_{n}=C^{0}\left([-c \tau, 0], \mathbf{R}^{n}\right)$.

The following are the main results of this section. For $\tau \geq 0$ there is a $c^{*}(\tau) \geq 0$ such that

1. $c^{*}$ is the minimal wave velocity, i.e. for $c<c^{*}$ there are only trivial waves, and for $c>c^{*}$ there are uniquely determined nontrivial wave solutions with distinct asymptotic behavior (Theorem 2.7); we will look at the stability of these solutions in $2.10,2.11$ and $\S 4$.

2. $c^{*}$ is the asymptotic speed of propagation for initial values with compact support (Theorem 2.12).

3. $c^{*}$ depends only on the time lag $\tau$ and the behavior of $f$ near 0 (Lemma 2.5).

For technical reasons it is often useful to rewrite the FDE (2.9) as

$$
u^{\prime}(s)=v(s), \quad v^{\prime}(s)=c v(s)-f_{c}\left(u_{s}\right)
$$

or-near $u=v=0$-as

$$
z^{\prime}(s)=L\left(z_{s}\right)+g\left(z_{s}\right), \quad z_{s} \in C_{2}=C^{0}\left([-c \tau, 0], \mathbf{R}^{2}\right),
$$

where $z=(u, v)^{t}$ is the transpose of $(u, v)$,

$$
L \phi:=\left(\begin{array}{l}
\phi_{2}(0) \\
c \phi_{2}(0)-f_{c}^{\prime}(0)\left(\phi_{1}\right)
\end{array}\right), \quad \phi \in C_{2},
$$

and $g \in C^{1, \alpha}\left(C_{2}, \mathbf{R}^{2}\right)$ with

$$
g(0)=g^{\prime}(0)=0
$$

The position of the spectrum of the infinitesimal generator $A$ of the semigroup generated by the linearized equation

$$
z^{\prime}(s)=L\left(z_{s}\right)
$$


on $C^{0}\left([-c \tau, 0], \mathbf{R}^{2}\right)$ is crucial for the existence of wave solutions and for the asymptotic speed for solutions of (2.1). $A: D(A) \subset C_{2} \rightarrow C_{2}$ is defined by

$$
A \phi(\theta)= \begin{cases}\phi^{\prime}(\theta) & \text { for }-c \tau \leq \theta<0 \\ L(\phi) & \text { for } \theta=0\end{cases}
$$

We have the following

2.4 LEMMA [16, pp. 168 and 177]. A has a pure point spectrum $\sigma(A)$, and $\lambda$ is a spectral value of $A$ iff $\Delta_{c}(\lambda)=0$ with

$$
\Delta_{c}(\lambda)=\lambda^{2}-c \lambda+\alpha+\beta e^{-\lambda c \tau}
$$

with $\alpha=\partial_{1} f(0,0) \geq 0, \beta=\partial_{2} f(0,0) \geq 0$.

The algebraic multiplicity of the eigenvalue $\lambda$ is equal to the multiplicity of the zero $\lambda$ of $\Delta_{c} . \sigma(A)$ is bounded to the right, and for every $\mu \in R$ there are at most finitely many $\lambda \in \sigma(A)$ with real parts greater than $\mu$.

The following properties of the characteristic function $\Delta_{c}$ (and of $\sigma(A)$ ) are needed.

2.5 LEMMA. (i) $\Delta_{c}$ is analytic on $\mathbf{C}$ and convex on $\mathbf{R}$; the number (2.19) $c^{*}:=\inf \left\{c>0 \mid\right.$ there is $s \in \mathbf{R}$ with $\left.\Delta_{c}(s)<0\right\}$ is positive, and

(a) $\Delta_{c}$ is positive on $\mathbf{R}$ for $c<c^{*}$.

(b) For $c>c^{*}$ there are exactly two real zeros $0<\lambda^{-}(c)<\lambda^{+}(c)<c$.

(c) For $c=c^{*}$ there is precisely one double zero $\left.\lambda^{*} \in\right] 0, c^{*}[$ on $R$.

(ii) $c^{*}$ is a smoothly decreasing function of $\alpha, \beta$ and $\tau$ respectively.

(iii) For $c>c^{*}, \lambda^{-}(c)$ decreases differentiably with $c$, and $\lambda^{-}(c) \rightarrow 0$ for $c \rightarrow \infty$.

(iv) For $c \geq c^{*}$, every nonreal zero of $\Delta_{c}$ has real part smaller than $\lambda^{-}(c)$ (resp. $\left.\lambda^{*}\left(c^{*}\right)\right)$.

(v) If $\beta>0$ then $c^{*}(\tau)$ and $\lambda^{*}(\tau)$ strictly decrease differentiably with $\tau$, and we have

$$
\begin{array}{llrl}
c^{*}(0) & =2 \sqrt{\alpha+\beta}, & & \frac{d}{d \tau} c^{*}(0)=-2 \beta \sqrt{\alpha+\beta}<0, \\
\lambda^{*}(0) & =\sqrt{\alpha+\beta}, & \frac{d}{d \tau} \lambda^{*}(0) & =0, \\
c^{*}(\infty) & =2 \sqrt{\alpha}, & \lambda^{*}(\infty) & =\sqrt{\alpha} .
\end{array}
$$

The verification of these properties involves more or less elementary calculations and is therefore omitted.

From the properties of $\sigma(A)$ in Lemma 2.4 we can describe the asymptotic behavior of solutions $z$ to the FDE (2.13) with $z(-\infty)=0$ : Let 0 be a hyperbolic critical point of $A$, i.e. there are no spectral points of $A$ on the imaginary axis. Let $\Lambda$ be the part of $\sigma(A)$ in the complex right half plane $\mathrm{C}^{+} ; \Lambda$ is finite and may consist of eigenvalues $\lambda_{1}, \ldots, \lambda_{k}$ with algebraic multiplicities $m_{i}=m\left(\lambda_{i}\right)$. Let $m=\sum m_{i}$ and let $\tilde{C}_{2}=C^{0}\left([-c \tau, 0], \mathbf{R}^{2}\right)$ be the natural domain of $L$ and $g$. Let $U \subset \tilde{C}_{2}$ be the generalized eigenspace to $\Lambda$, i.e. $U$ is spanned by the functions

$$
\phi_{i j}(\theta)=\gamma_{i j} \theta^{j-1} e^{\lambda_{i} \theta}, \quad 1 \leq i \leq k, 1 \leq j \leq m_{i},
$$

with suitable $\gamma_{i j} \in \mathbf{R}^{2}$ [16, pp. 175-177]. Let $\pi$ be a projection of $\tilde{C}_{2}$ on $U$ as in $[16$, p. 186] and $y$ a solution of the linearized equation $(2.16)$ with $y(-\infty)=0$, 
let $Y(s) \in \mathbf{R}^{m}$ be the coefficient vector of $y_{s}=\pi\left(y_{s}\right) \in U$ with respect to the basis $\left\{\phi_{i j}\right\}$. Then there is a matrix $B \in \mathbf{R}^{(m, m)}$ with $\sigma(G)=\Lambda$ (with the resp. multiplicities) such that

$$
Y^{\prime}(s)=B Y(s) \text {. }
$$

A function $\varphi: \mathbf{R} \rightarrow \mathbf{R}^{m}$ has exact order $(\mu, r),\left(\mu\right.$ real, $\left.r \in \mathbf{N}_{0}\right)$ for $s \rightarrow-\infty$ iff

$$
\begin{aligned}
0 & <\liminf _{s \rightarrow-\infty} s^{-r} e^{-\mu s}|\varphi(s)| \\
& \leq \limsup _{s \rightarrow-\infty} s^{-r} e^{-\mu(s)}|\varphi(s)|<\infty ;
\end{aligned}
$$

obviously every solution $Y(s)$ of $(2.21)$ has an exact order $(\mu, r)$ with $\mu=\operatorname{Re}(\lambda)$ for some $\lambda \in \Lambda$ and $0 \leq r<m(\lambda)$, and for every such pair $(\mu, r)$ there is a solution $Y$ of $(2.21)$ with this given exact order. Since $Y(s)$ is the coefficient vector of $y_{s}$, the same results hold for solutions $y$ of (2.16). These notations and preliminaries are prerequisites for the following result.

2.6 Proposition. Let $z$ be a solution of the nonlinear FDE (2.13) with $z(-\infty)$ $=0$. Then there is a solution $y$ of the linearized equation (2.16) with exact order $(\mu, r)$ depending on $z$, such that

$$
z(s)=y(s)(1+O(1)) \quad \text { for } s \rightarrow-\infty .
$$

On the other hand, for every solution $y$ of (2.16) with exact order $(\mu, r)$ there is a solution $z$ of (2.13) fulfilling (2.23). If $\mu=\max \{\operatorname{Re} \lambda \mid \lambda \in \Lambda\} z$ is uniquely determined by $y$.

ProOF. Let $z(\phi)$ denote the solution of (2.13) with initial value $\phi \in C_{2}$. For small $\varepsilon>0$, the set

$$
\mathcal{U}_{\varepsilon, g}:=\left\{\phi \in C_{2} \mid\|\phi\|<\varepsilon, z(\phi) \text { bounded on } \mathbf{R}^{-}\right\}
$$

is diffeomorphic to $U \cap\{\|\phi\|<\varepsilon\}$ [16, Theorem 10.1.11]. $U_{\varepsilon, g}$ is tangential to $U$ in 0 , i.e.

$$
\lim \|(1-\pi) \phi\| /\|\pi \phi\|=0 \quad \text { for }\|\phi\| \rightarrow 0, \phi \in \mathcal{U}_{\varepsilon, g}
$$

$\pi$ is again the projection of $C_{2}$ onto $U . \pi$ restricted to $U_{\varepsilon, g}$ is a diffeomorphism between $\mathcal{U}_{\varepsilon, g}$ and $U \cap\{\|\phi\|<\varepsilon\}$. Furthermore there are positive constants $M$ and $\gamma$ such that

$$
\left\|z_{s}(\phi)\right\| \leq M e^{\gamma s} \text { for } s<0, \phi \in \mathcal{U} .
$$

So for sufficiently small initial values $\phi$ we may consider the ordinary differential equation

$$
Z^{\prime}(s)=B Z(s)+G(Z(s)), \quad Z(-\infty)=0,
$$

instead of the FDE (2.13); $Z(s)=\operatorname{Coeff}\left(\pi\left(z_{s}\right)\right) \in \mathbf{R}^{m}, B$ is taken from (2.21), and $G(Z(s))$ behaves like $g\left(z_{s}\right)$. From (2.22) and (2.25) we get

$$
\lim _{s \rightarrow-\infty} \inf \frac{\log |Z(s)|}{s}=\mu \geq \gamma>0
$$


and $g$ and $G$ are regular enough so that theorems $[6,13.4 .3,13.4 .5]$ on the asymptotic behavior of ODEs give the desired results for $Z(s)=\operatorname{Coeff}\left(\pi\left(z_{s}\right)\right)$ and the solution $Y(s)=\operatorname{Coeff}\left(y_{s}\right)$ of $(2.21)$. The decomposition $(y-z)_{s}=\left(y_{s}-\pi\left(z_{s}\right)\right)+\left(\pi\left(z_{s}\right)-\right.$ $z_{s}$ ) and the tangency condition (2.24) complete the proof of the theorem.

After these investigations on the asymptotic behavior of solutions to FDEs we prove the following theorem on the existence of solutions for the wave equation

$$
u^{\prime \prime}-c u^{\prime}+f_{c}\left(u_{s}\right)=0 \text {. }
$$

2.7 THEOREM. Let $c^{*}$ be given as in Lemma 2.5. $c^{*}$ is the minimal speed to equation (2.9), i.e.

(i) there are only trivial wave solutions to (2.9) for $0 \leq c<c^{*}$, and

(ii) for all $c>c^{*}$ there are nontrivial wave solutions $u_{c}$ of (2.9) with the following asymptotic behavior. There are positive constants $K_{1}(c), K_{2}(c)$ such that

$$
\begin{aligned}
& u_{c}^{(n)}(s)=K_{1} \lambda^{-}(c)^{n} e^{\lambda^{-}(c) s}(1+o(1)) \quad \text { for } s \rightarrow-\infty, \\
& \left(u_{c}-1\right)^{(n)}(s)=-K_{2} \mu(c)^{n} e^{\mu(c) s}(1+o(1)) \text { for } s \rightarrow+\infty
\end{aligned}
$$

for $0 \leq n \leq 3 ; \lambda^{-}(c)$ is the smaller positive zero of $\Delta_{c}$,

$$
\mu(c)=\frac{1}{2}\left(c-\left(c^{2}-4 \partial_{1} f(1,1)\right)^{1 / 2}\right)
$$

the negative zero of the linearization of (2.9) around the trivial solution $u=1$.

PROOF. (i) follows directly from Proposition 2.6 since there are only complex eigenvalues, i.e. oscillating solutions for $s \rightarrow-\infty$ for $c<c^{*}$.

(ii) The existence of wave solutions and their asymptotic behavior for $s \rightarrow-\infty$ can be shown e.g. by the following Theorem 2.9 using the sub- and supersolutions already given by Atkinson and Reuter [4]. Schumacher's proof of existence and uniqueness (up to translation) of wave solutions to certain integrodifferential equations $[30,31]$ can be carried over precisely to our case. The asymptotic behavior for $s \rightarrow+\infty$ can be shown as in the "cubic" case in the next paragraph (Corollary $3.15)$.

Furthermore it is very elementary to show that every nontrivial wave solution $u$ to (2.9) must be strictly monotone, take values strictly between 0 and 1 and take the limits $u(-\infty)=0, u(+\infty)=1$.

In the following we show that $c$ is also the asymptotic speed of propagation under equation (2.1) of initial values that have compact support or decay quickly enough. For this purpose we define

2.8 Definition. Let $f$ satisfy Hypotheses 2.1 , and let $f_{c}$ be given by 2.11 . A bounded continuous function $\varphi: \mathbf{R} \rightarrow \mathbf{R}$ is called a subsolution (or supersolution respectively) to

$$
u^{\prime \prime}-c u^{\prime}+f_{c}\left(u_{s}\right)=0
$$

iff there are twice continuously differentiable functions $\psi_{1}, \ldots, \psi_{n}: \mathbf{R} \rightarrow \mathbf{R}$ with $\varphi=\max \left(\psi_{1}, \ldots, \psi_{n}\right)\left(\right.$ or $\left.\varphi=\min \left(\psi_{1}, \ldots, \psi_{n}\right)\right)$ and for every $s \in \mathbf{R}, 1 \leq i \leq n$, we have

$$
\varphi(s)=\psi_{i}(s) \Rightarrow \psi_{1}^{\prime \prime}(s)-c \psi_{i}^{\prime}(s)+f_{c}\left(\varphi_{s}\right) \geq 0 \quad(\text { or } \leq 0) .
$$

The main tool for the subsequent investigations is the following theorem which can be found in [2] for parabolic equations without deviating arguments. 
2.9 PROPOSITION. Let $f$ satisfy Hypotheses $2.1, \varphi \in B C^{0}(\mathbf{R}, \mathbf{R})$ be a subsolution (or supersolution, respectively) to (2.9), and $0 \leq \varphi \leq 1$.

Let $v$ be a solution of the Cauchy problem

$$
\begin{gathered}
\left(\partial_{t}+c \partial_{s}-\partial_{s}^{2}\right) v(s, t)=F_{c}(v)(s, t) \quad \text { for } s \in \mathbf{R}, t>0 \\
v(\cdot, t)=\varphi \quad \text { for } t \leq 0
\end{gathered}
$$

with

$$
F_{c}(v)(s, t):=f(v(s, t), v(s-c \tau, t-\tau)) .
$$

Then $v(\cdot, t)$ converges monotonically increasing (decreasing) towards the minimal (maximal) wave solution $q$ of (2.9) with $q \geq \varphi(q \leq \varphi)$ on $\mathbf{R}, 0 \leq q \leq 1$.

The proof is given for the subsolutions; obviously the other case is analogous. It is performed in several steps: The crucial estimate is

$$
v \geq \varphi \text { for all } t \geq 0 .
$$

The proof of (2.33) is rather technical: Setting $w:=\varphi-v$ we obviously have

$$
w(\cdot, t) \leq 0 \text { for } t \leq 0,-1 \leq w \leq 1 \text { on } \mathbf{R} \times \mathbf{R}^{+} .
$$

With $w^{+}:=\max (w, 0)$ and

$$
\left\|w^{+}\right\|_{t}:=\sup \left\{w^{+}(s, r) \mid s \in \mathbf{R}, r \leq t\right\},
$$

" $v \geq \varphi$ " is equivalent to " $\left\|w^{+}\right\|_{t} \leq 0$ ". Let $T>0$ and

$$
\sigma(s, t):=e^{\mu t}\left\{\varepsilon\left(1+\log \left(1+s^{2}\right)\right)+\gamma(t)\right\}
$$

where $\mu>2+c>0$ is a constant and $\gamma \in C^{1}([0, T], \mathbf{R})$ an arbitrary function with

$$
\gamma(0)=\left\|w^{+}\right\|_{0}=0, \quad\left\|w^{+}\right\|_{t} \leq \gamma^{\prime}(t) / K \leq 1 \quad \text { for } 0 \leq t \leq T
$$

$K$ being a positive constant with

$$
K>\sup \left\{\left|\partial_{1} f(r, s)\right|+\left|\partial_{2} f(v, s)\right| \mid r, s \in \mathbf{R}\right\} .
$$

In the following we show that

$$
0 \leq\left\|w^{+}\right\|_{t} \leq e^{\mu T} \gamma(t) \text { for } 0 \leq t \leq T,
$$

and from this follows (Hilfssatz 1 from [26, p. 164])

$$
\left\|w^{+}\right\|_{t}=0 \text { for } 0 \leq t \leq T
$$

this is equivalent to the desired result (2.33).

To prove (2.39), we claim that

$$
w(s, t)<\sigma(s, t) \text { for all } s \in \mathbf{R}, 0 \leq t \leq T .
$$

Suppose (2.40) does not hold. Then there is a "first" point $P:=\left(s_{0}, t_{0}\right), 0<t_{0} \leq T$, with $w(s, t)<\sigma(s, t)$ for $s \in \mathbf{R}, t<t_{0}$, and

$$
w(P)=\sigma(P) .
$$

It is elementary to prove that $\varphi$ is continuously differentiable in $s_{0}$, the unilateral derivatives $\varphi^{\prime \prime}\left(s_{0}+\right), \varphi^{\prime \prime}\left(s_{0}-\right)$ exist and fulfil

$$
\min \left(\varphi^{\prime \prime}\left(s_{0}+\right), \varphi^{\prime \prime}\left(s_{0}-\right)\right) \leq \partial_{s}^{2}(\sigma+v)(P) .
$$


So the following relations are valid in $P$.

$$
\begin{aligned}
& \partial_{t} W(P) \geq \partial_{t} \sigma(P)=\mu \sigma(P)+e^{\mu t_{0}} \gamma^{\prime}\left(t_{0}\right)>0 \\
& \partial_{s} W(P)=\partial_{s} \sigma(P)=2 \varepsilon e^{\mu t_{0}} s_{0} /\left(1+s_{0}^{2}\right)
\end{aligned}
$$

and in particular

$$
\left|\partial_{s} \sigma(P)\right| \leq \varepsilon e^{\mu t_{0}} \leq \sigma(P),
$$

and from (2.42) one obtains

$$
\partial_{s}^{2} w\left(s_{0} \pm, t_{0}\right) \leq \partial_{s}^{2} \sigma(P)=2 e^{\mu t_{0}}\left(1-s_{0}^{2}\right) /\left(1+s_{0}^{2}\right)^{2} \leq 2 \sigma(P) .
$$

$\varphi$ being a subsolution, we have

$$
\varphi^{\prime \prime}\left(s_{0} \pm\right)-c \varphi^{\prime}\left(s_{0}\right) \geq-f_{c}\left(\varphi_{s_{0}}\right) .
$$

Combining inequalities (2.43)-(2.46), the choice (2.38) of $K,(2.47),(2.41)$ and the quasimonotonicity of $f$, we obtain

$$
\begin{aligned}
\left(\partial_{t}-\partial_{s}^{2}+c \partial_{s}\right) \sigma(P) \leq & \left(\partial_{t}-\partial_{s}^{2}+c \partial_{s}\right) w\left(s_{0} \pm, t_{0}\right) \\
\leq & f_{c}\left(\varphi_{s_{0}}\right)-F_{c}(v)(P) \\
= & f_{c}\left(\varphi_{s_{0}}\right)-F_{c}(\max (\varphi, v))(P) \\
& +F_{c}(\max (\varphi, v))(P)-F_{c}(v)(P) \\
\leq & K\left\|w^{+}\right\|_{t_{0}} .
\end{aligned}
$$

However, by direct estimation we obtain

$$
\begin{array}{r}
\left(\partial_{t}-\partial_{s}^{2}+c \partial_{s}\right) \sigma(P) \geq\left(\partial_{t}-\partial_{s}^{2}\right) \sigma(P)-c\left|\partial_{s} w(P)\right| \\
\geq \mu \sigma(P)+e^{\mu t_{0}} \gamma^{\prime}\left(t_{0}\right)-(2+c) \sigma(P) \geq \gamma^{\prime}\left(t_{0}\right)
\end{array}
$$

(2.48) and (2.49) together are a contradiction to (2.45), so (2.39) holds, and (2.33) is proved. To prove the uniform convergence of $v(\cdot, t)$ for $t \rightarrow \infty$, we note that

(2.50) $v(s, \cdot)$ is monotonically increasing for all $s \in \mathbf{R}$ and

(2.51) The set $\{v(\cdot, t) \mid t \geq 2\}$ is equicontinuous.

(2.50) and (2.51) can be proved as in [2].

With (2.50) and (2.51), the Arzela-Ascoli theorem yields monotonic locally uniform convergence of $v(\cdot, t)$ to a bounded continuous function $q$ with $0 \leq \varphi \leq q \leq 1$ on $\mathbf{R}$; one can verify (e.g. $[13$, pp. $8-13]$ that $q$ is the minimal solution $\geq \varphi$ of the wave equation (2.9).

We use this theorem to prove the following result.

2.10 THEOREM. Let $c>c^{*}$. Then there is a wave solution $u_{c}$ with the asymptotic behavior (2.27), and (2.27) is the only possible behavior of nontrivial wave solutions for $s \rightarrow-\infty$. The wave solution $u_{c}$ is unstable in the following sense.

(i) Let $0 \leq \bar{\varphi} \leq 1$ be a supersolution of (2.9) with $\bar{\varphi}(s)=1$ for all $s \geq s_{0} \in \mathbf{R}$, and with the asymptotic behavior

$$
\lim _{s \rightarrow-\infty} \bar{\varphi}(s) / u_{c}(s)=0 .
$$

If $v$ is a solution to (2.30) with initial values $v(\cdot, t) \leq \bar{\varphi}$ for $t \leq 0$, then

$$
\lim _{t \rightarrow \infty} v(\cdot, t)=0 \quad \text { uniformly on compact intervals. }
$$


(ii) Let $0 \leq \underline{\varphi} \leq 1$ be a supersolution of (2.9) with

$$
\lim _{s \rightarrow-\infty} \underline{\varphi}(s) / u_{c}(s)=\infty .
$$

If $v$ is a solution to (2.30) with initial values $\underline{\varphi} \leq v(\cdot, t) \leq 1$, for $t \leq 0$, then

$$
\lim _{t \rightarrow \infty} v(\cdot, t)=1 \quad \text { uniformly on compact intervals. }
$$

PROOF. Using the supersolution $\bar{\varphi}(s)=\min \left(1, \exp \left(\lambda^{-}(c) \cdot s\right)\right)$ and the subsolution $\underline{\varphi}(s)=\max \left(0,(1-M \exp (\varepsilon s)) \cdot \exp \left(\lambda^{-}(c) \cdot s\right)\right)$ with $c>0$, with $M$ sufficiently large [4], Theorem 2.9 delivers wave solutions with the asymptotic behavior (2.27). According to Theorem 2.7 and the properties of the characteristic equation $\Delta_{c}$ (Lemma 2.5(i)), every nontrivial wave solution $u$ exhibits the asymptotic behavior (2.27) or

$$
\left(u(s), u^{\prime}(s)\right) \sim e^{\lambda^{+}(c) s}\left(1, \lambda^{+}(c)\right) \text { for } s \rightarrow-\infty .
$$

Using phase plane arguments as in $\S 3$ it is easy to prove that every positive solution satisfying (2.56) will reach the value $u=1$ in some points $s_{0} \in R$ with a positive slope $u^{\prime}\left(s_{0}\right)$, so it is no wave solution in the sense of Definition 2.3.

This proves the instability criteria (i) and (ii) since the limiting traveling waves obtained by Theorem 2.9 must be the trivial solutions 0 and 1 due to conditions (2.52) resp. (2.54); the maximum principle completes the proof.

2.11 REMARKS. Theorem 2.10 is an instability criterion for the wave solution $u_{c}$ in the following sense. The solution $\varphi(s):=\min \left(u_{c}(s), \exp \left(\lambda\left(s-s_{0}\right)\right)\right)$ with $\lambda \in] \lambda^{-}(c), \lambda^{-}(c)$ [ is a supersolution to (2.9) with the asymptotic behavior (2.52). Choosing $s_{0}$ appropriately, the norm of $u_{c}-\varphi$ may be arbitrarily small in every $L^{p}(R), 1 \leq p \leq \infty$, and yet we have the unstable behavior (2.53). So together with the "dual" property 2.10 (ii) we have the following necessary stability criterion: If a solution $v$ of (2.30) with initial value $v_{0}$ converges uniformly to the traveling wave $u_{c}$ for $t \rightarrow \infty$, then we have

$$
0<\lim _{\substack{s \rightarrow-\infty \\-\tau \leq t \leq 0}} \inf \frac{v_{0}(s, t)}{u_{c}(s)} \leq \lim _{\substack{s \rightarrow-\infty \\-\tau \leq t \leq 0}} \sup \frac{v_{0}(s, t)}{u_{c}(s)}<\infty .
$$

Stability of $u_{c}$ will be investigated somewhat closer in $\S 4$. For equations without delay, criterion 2.10(i) can be found in [19]. One can show the continuous dependence of the wave solutions on $c$ and $\tau$ in the sense of Theorem 3.16; the proof is omitted here.

The following theorem shows that $c^{*}$ is also the asymptotic speed of propagation in the sense of Aronson-Weinberger:

2.12 THEOREM. Let $f$ satisfy conditions 2.1 , and $w$ be a solution to the original Cauchy problem (2.1-2) with continuous initial function $0 \leq w_{0} \leq 1$, supp $w_{0}$ compact in $\mathbf{R} \times[-\tau, 0]$, and $w_{0}(\cdot, 0) \not \equiv 0$. Then for all $x \in \mathbf{R}$ we have

$$
\begin{array}{ll}
\lim _{t \rightarrow \infty} w(x-c t, t)=0 & \text { for }|c|>c^{*}, \\
\lim _{t \rightarrow \infty} w(x-c t, t)=1 & \text { for }|c|<c^{*} .
\end{array}
$$

PROOF. In analogy to [2] it is enough to consider the case $c \geq 0$. For $c>c^{*}$ we have $w_{0}(\cdot, t) \leq \bar{\varphi}$ for the supersolution $\bar{\varphi}(s)=\min \left(1, \exp \left(\lambda^{+}(c)\left(s-s_{0}\right)\right)\right)$ with an appropriate $s_{0} \in \mathbf{R}$, and (2.58) follows from Theorem 2.10(i). 
Now let $0 \leq c<c^{*}$. For $c=0$, Kobayashi [23] shows

$$
\lim _{t \rightarrow \infty} w(\cdot, t)=1 \quad \text { uniformly on compact intervals. }
$$

In 2.13 we construct a subsolution $\varphi$ of $(2.9)$ with compact support and

$$
0 \leq \varphi \leq \varepsilon<1 \text {. }
$$

Let $v(s, t):=w\left(s+c t, t+t_{0}\right)$. Because of $(2.60)$ there is a $t_{0}$ such that $v(\cdot, t) \geq \varphi$ for $-\tau \leq t \leq 0$. $v$ satisfies the transformed equation (2.7). If $\underline{v}$ is a solution to (2.7) with initial value $\varphi$, then by Theorem $2.9, \underline{v}(\cdot, t)$ converges monotonically to 1 since there are no nontrivial wave solutions for $c<c^{*}$, and the maximum principle proves (2.59) provided we know a suitable subsolution.

2.13 Constructing a subsolution for $0<c<c^{*}$. Let $c<c^{*}$ be given. Because of hypotheses 2.1 on $f$ and Lemma 2.5 (ii) there is $\varepsilon>0$ and $\gamma=\gamma(\varepsilon) \in] 0,1[$ with

$$
\gamma(\alpha r+\beta t)<f(r, t) \text { for all } 0<r, t<\varepsilon,
$$

and at the same time there is no real zero of

$$
\tilde{\Delta}_{c}(\lambda)=\lambda^{2}-c \lambda+\gamma \alpha+\gamma \beta e^{-c \tau \lambda},
$$

the characteristic function of

$$
v^{\prime \prime}(s)-c v^{\prime}(s)+\gamma \alpha(s)+\gamma \beta v(s-c \tau)=0 .
$$

Setting $u(s)=v(s) e^{-c s}$ and $w=u^{\prime}+c u$, then (2.63) is equivalent to

$$
u^{\prime}(s)=w(s)-c u(s), \quad w^{\prime}(s)=-\rho u(s)-\eta u(s-c \tau)
$$

with $\rho=\gamma \alpha, \eta=\gamma \beta e^{-c \tau}$.

Choose an initial value $\left(u_{0}, a\right) \in C^{2}([-\tau, 0], \mathbf{R}) \times \mathbf{R}^{+}$with $a>0, u_{0}(0)=0$, $u_{0}^{\prime}(0)=a$, and $u_{0}^{\prime \prime}(0)=-c a-\eta u_{0}(-c \tau)$. Then it is easy to show that there is $s_{1}>0$ with $u^{\prime}\left(s_{1}\right)=0$ and $s_{2}>s_{1}$ with $u\left(s_{2}\right)=0, u>0$ on $] 0, s_{2}[$. The solution $v(s)=u(s) e^{c s}$ of $(2.63)$ on $\left[0, s_{2}\right]$ has the same qualitative behavior. If we choose $\delta$ small enough such that $\delta v<\varepsilon$ on $\left[0, s_{2}\right]$, then it is easy to verify that $\varphi:=\max (0, \delta v)$ is a subsolution to $(2.9)$ with the desired properties.

3. Nonlinearities changing sign. If $f$ admits an intermediate steady state, one cannot in general construct subsolutions (cf. e.g. [12]). Sometimes however it is possible to use phase arguments as in the case without time delay. First of all we list the hypotheses for $f$ used in this paragraph.

3.1 Hypotheses. Let the nonlinearity $f$ satisfy the following hypotheses.

(i) $f \in C^{1, \nu}\left([0,1]^{2}, \mathbf{R}\right), f(0,0)=f(1,1)=0, \partial_{2} f(r, t)>0$ for $0<r<1$ (strict quasimonotonicity), $\partial_{2} f\left(r, t_{1}\right) \leq \partial_{2} f\left(r, t_{2}\right)$ for $0<r<1,0<t_{1}<t_{2}<1$ ("convexity").

(ii) There is an intermediate zero $a \in] 0,1[$ with $f(a, a)=0, f(r, t)<0$ for $0<r$, $t<a, f(r, t)>0$ for $a<r, t<1$.

(iii) $\partial_{1} f(0,0)=-\alpha<0, \partial_{1} f(1,1)=-\beta<0, \partial_{2} f(0, \cdot) \equiv 0$.

3.2 REMARKS. (i) Hölder continuity of the derivatives is only used for determining the asymptotic behavior of the traveling waves (Corollary 3.15); otherwise, $f$ has to be continuously differentiable only. 
(ii) Let $f$ satisfy 3.1 , and set $f_{0}(r):=f(r, r) . f$ can be extended to a function $f \in C^{1}\left(\mathbf{R}^{2}, \mathbf{R}\right)$ such that

$$
f(r, t)=f_{0}(r) \begin{cases}>0 & \text { for } r<0, t \in \mathbf{R} \\ <0 & \text { for } r>1, t \in \mathbf{R} .\end{cases}
$$

(iii) An example for a nonlinearity satisfying 3.1 is given by

$$
f(r, t):= \begin{cases}r(1-r)(t-a) & \text { for } 0 \leq r \leq 1, t \in \mathbf{R}, \\ r(1-r)(r-a) & \text { otherwise }\end{cases}
$$

with $0<a<\frac{1}{2}$ and without time delay $(\tau=0)$ this is the Huxley nonlinearity.

Under Hypotheses 3.4, we are again interested in wave solutions to (2.1), i.e. stationary solutions to

$$
\partial_{t} W+c \partial_{s} W-\partial_{s}^{2} W=f(W(s, t), W(s-c \tau, t-\tau)) .
$$

Simple differential inequalities and the maximum principle 2.2 yield the following stability result for the "trivial" constant wave solutions.

3.3 LEMMA. (i) The steady state solutions $W \equiv 0$ and $W \equiv 1$ are stable in the following sense:

(a) If $0 \leq W \leq \bar{w}_{0}$ for $s \in \mathbf{R},-\tau \leq t \leq 0$, with $\left.\bar{w}_{0} \in\right] 0$, a[, then $W$ converges to 0 uniformly on $\mathbf{R}$ for $t \rightarrow \infty$.

(b) If $w_{0} \leq W \leq 1$ for $s \in \mathbf{R},-\tau \leq t \leq 0$, with $\left.w_{0} \in\right] a, 1$ [, then $W$ converges to 1 uniformly on $\mathbf{R}$ for $t \rightarrow \infty$.

(ii) The intermediate steady state $W \equiv a$ is unstable.

The wave solutions $u, 0 \leq u \leq 1$, to (2.1) satisfy the ordinary functional differential equation (FDE)

$$
\ddot{u}(s)-c \dot{u}(s)+f(u(s), u(s-c \tau))=0, \quad s \in \mathbf{R},
$$

hence the equivalent system

$$
\dot{u}=v, \quad \dot{v}=c v-f(u, u(\cdot-c \tau)) .
$$

Furthermore, in this section we require the asymptotic boundary conditions

$$
u(-\infty)=v(-\infty)=0
$$

The main result of this section is the following (see Theorems 3.13 and 3.16). For every $\tau \geq 0$ there is exactly one $c^{*}(\tau)$ and one unique (up to translation) wave solution $u$ of (3.3). $c^{*}$ and $u$ satisfy

$-0<u<1$ on $\mathbf{R}$,

- $u$ is strictly monotonically increasing,

- $u$ and $c^{*}$ depend continuously on $\tau$,

$-\operatorname{sign} c^{*}(\tau)=\operatorname{sign} \int_{0}^{1} f_{0}(r) d r$.

In this section we often compare solutions to (3.2) or (3.3) with solutions of the ordinary differential equation

$$
\ddot{\varphi}(s)-c \dot{\varphi}(s)+f_{0}(\varphi(s))=0, \quad s \in \mathbf{R},
$$

or

$$
\begin{aligned}
& \dot{\varphi}=\psi, \quad \dot{\psi}=c \psi-f_{0}(\varphi) \\
& \varphi(-\infty)=\psi(-\infty)=0
\end{aligned}
$$


The linearizations of (3.3) and (3.6) around $(0,0)$ have

$$
\lambda(c)=c / 2+\sqrt{c^{2} / 4+\alpha}, \quad \alpha=-\partial_{1} f(0,0)>0,
$$

as their only eigenvalue in $\mathbf{C}_{0}^{+}$, and correspondingly

$$
\mu(c)=c / 2-\sqrt{c^{2} / 4+\beta}, \quad \beta=-\partial_{1} f(1,1)>0,
$$

is the only eigenvalue in $\mathbf{C}_{0}^{-}$of the linearizations of (3.3) and (3.6) around $(1,0)$. So candidates for wave solutions to (3.2) and (3.5) have the same asymptotic behavior for $s \rightarrow-\infty[\mathbf{1 6}$, Chapter 10, and 6, Chapter 15]:

3.4 LEMMA. Each of equations (3.3), (3.4) and (3.6), (3.7) have-up to translation-exactly one solution which is positive for sufficiently large negative s; these solutions satisfy

$$
u(s)=v(s)=\lambda(c) u(s)+o(u(s)) \quad \text { for }(3.3),(3.4) \quad \text { as } s \rightarrow-\infty
$$

and

$$
\varphi(s)=\psi(s)=\lambda(c) \varphi(s)+o(\varphi(s)) \quad \text { for }(3.6),(3.7) \quad \text { as } s \rightarrow-\infty .
$$

In the following we are only interested in positive solutions $u$ and $\varphi$ and follow them only as long as they are monotonically increasing. As long as $\dot{\varphi} \geq 0$, we have for the trajectory $\Psi(\eta):=\varphi^{\prime}\left(\varphi^{-1}(\eta)\right)$ the graph equation in the $(\varphi, \dot{\varphi})$-phase space:

$$
\Psi^{\prime}(\eta)=c-f_{0}(\eta) / \Psi(\eta) \quad \text { for } \eta>0 .
$$

(In this section $/$ denotes the derivatives $d / d \eta$.)

For the solution of the graph equation (3.12) corresponding to the trajectory for (3.6-7), equations (3.7) and (3.11) give the initial values

$$
\Psi(0+)=0, \quad \Psi^{\prime}(0+)=\lambda(c) .
$$

In the following investigations we make extensive use of the graph equation for trajectories of the FDE (3.3); for $V(\eta)=v\left(u^{-1}(\eta)\right)(\eta=u(s))$ holds

$$
V^{\prime}(\eta)=c-f\left(\eta, \eta_{c \tau}\right) / V(\eta)
$$

$\eta_{c \tau}$ is given implicitly by the relation

$$
\int_{\eta_{\mathrm{c} \tau}}^{\eta} \frac{d \sigma}{V(\sigma)}=c \cdot \tau .
$$

Again, the asymptotic boundary conditions (3.4) and Lemma 3.4 provide

$$
V(0+)=0, \quad V^{\prime}(0+)=\lambda(c) .
$$

Since $\eta_{c \tau}$ cannot be given explicitly in terms of $V$ and $\eta$, working with the graph equation (3.14) for $V$ involves continuous "switching" between (3.14) and the original FDE (3.2) or (3.3). So first of all we give some general properties of trajectories $V(\eta)$ to $(3.3)$ with $V(0+) \geq 0$ that will be frequently used in the following proofs.

3.5 Lemma. Let $c \geq 0,0<a<1$, the intermediate zero of $f$. Then the following hold.

(i) $V(\eta)>0$ and $V^{\prime}(\eta) \geq c \geq 0$ for $0<\eta \leq a$,

(ii) $V^{\prime}(\eta) \geq c \geq 0$ for $\eta>1$. 
(iii) If there is a $\bar{\eta}>0$ with $V(\bar{\eta})=0$, then $a<\bar{\eta} \leq 1$.

(iv) If this $\bar{\eta}$ is smaller than 1 , then $\bar{\eta}$ is a local maximum of $u$, and $V(\eta) /(\eta-\bar{\eta}) \rightarrow-\infty$ for $\eta \uparrow \bar{\eta}$.

The next two lemmas compare solutions of (3.3), (3.4) and (3.14), (3.15) as well as their trajectories belonging to different velocities $c$ and $\tilde{c}$. Here $u, v$, and $V$ denote solutions for $c$; $\tilde{u}, \tilde{v}$ and $\tilde{V}$ denote solutions for $\tilde{c}$.

3.6 LEMMA. Let $0<\eta_{0}<1, \tilde{V}\left(\eta_{0}\right) \geq V\left(\eta_{0}\right)>0, \tilde{V}(\eta)>V(\eta)>0$ for $\eta<\eta_{0}$ (as long as $V(\eta)$ is defined). Then the following holds. If $\tilde{u}(0)=u(0)=\eta_{0}$, then $\tilde{u}(t)<u(t)$ for all $t<0$.

PROOF. If $\tilde{V}\left(\eta_{0}\right)>V\left(\eta_{0}\right)$, the proof is elementary. If $\tilde{V}\left(\eta_{0}\right)=V\left(\eta_{0}\right)$, take $\eta<\eta_{0}$. Approximate $u$ by $u_{\eta}(s):=u(s-m(\eta))$, and $\tilde{u}$ by $\tilde{u}_{\eta}(s):=\tilde{u}(s-\tilde{m}(\eta))$ such that $u_{\eta}(0)=\tilde{u}_{\eta}(0)=\eta$. Since $m(\eta)=\int_{\eta}^{\eta_{0}} d \sigma / V(\sigma)(\tilde{m}$ correspondingly $)$ and $\tilde{V}(\eta)>V(\eta)>0$ for $\eta<\eta_{0}$, the result holds for $u_{\eta}$ and $\tilde{u}_{\eta}$. Approximation for $\eta \uparrow \tilde{\eta}$ yields the weak inequality $\tilde{u} \leq u$ for $s<0$, but since an equality in some $s<0$ would give a contradiction, the proof is completed.

3.7 Lemma. Let $\tilde{c}>c \geq 0, \tilde{V}(0)=V(0)=0$. Then the trajectories $\tilde{V}$ and $V$ do not intersect in the first quadrant, i.e. more precisely

(i) $\tilde{V}(\eta)>V(\eta)$ if $V(\eta)>0$.

(ii) If $\tilde{V}(\tilde{\eta})=V(\eta)=0$ for values $\eta, \tilde{\eta} \in] 0,1[$, then $\tilde{\eta}>\eta$.

ProOF. (i) Because of $\lambda(\tilde{c})>\lambda(c)$ and Lemma 3.3 there is $\eta_{0}>0$ with $\tilde{V}(\eta)>$ $V(\eta)>0$ for $0<\eta<\eta_{0}$. Suppose there is a first intersection in $\left(\eta_{0}, \varsigma_{0}\right)$ with $\varsigma_{0}=\tilde{V}\left(\eta_{0}\right)=V\left(\eta_{0}\right)>0$, then we have

$$
\tilde{V}^{\prime}\left(\eta_{0}\right) \leq V^{\prime}\left(\eta_{0}\right) \text {. }
$$

Without restriction we can assume $\tilde{u}(0)=u(0)=\eta_{0}, \tilde{v}(0)=v(0)=\varsigma_{0}$. Using the graph equation (3.14) and noting that $\tilde{c}>c, \varsigma_{0}>0$. (3.17) is equivalent to

$$
0<\varsigma_{0}(\tilde{c}-c) \leq f\left(\eta_{0}, \tilde{u}(-\tilde{c} \tau)\right)-f\left(\eta_{0}, u(-c \tau)\right) .
$$

Since $f$ is strictly quasimonotonic and $u$ is strictly monotonically increasing on $\mathbf{R}^{-}$, we obtain

$$
\tilde{u}(-\tilde{c} \tau)>u(-c \tau)>u(-\tilde{c} \tau)
$$

this is a contradiction to Lemma 3.6. (ii) can be proved analogously to the second case in Lemma 3.6.

The next two theorems give more detailed information about the shape of trajectories $V$ in phase space. The first is used to prove continuous dependence of the trajectories on the velocity $c$ (Theorem 3.10). For $c \geq 0$ it compares the trajectories $V(\eta)$ of the FDE (3.3) with the trajectories $\Psi(\eta)$ of the corresponding ODE (3.6). For $c=0$, the FDE (3.3) degenerates to (3.6), so for $c=0$ we have the classical phase plane portrait for the Huxley-equation whose behavior for increasing $c$ is well known.

3.8 THEOREM. Let $c, \tau>0,(u, v)$ solution to $(3.3-4),(\varphi, \Psi)$ solution to (3.67), $V(\eta), \Psi(\eta)$ the corresponding trajectories with $V(0+)=\Psi(0+)=0$. Then

(i) $V(\eta)>\Psi(n)$ for all $\eta>0$ as long as $\Psi(\eta) \geq 0$. 
(ii) For all $\eta$ with $V^{\prime}(\eta) \geq 0$, especially for $\eta \in[0, a]$ holds

$$
V(\eta)<\Psi(\eta)+c \tau \rho(n) \quad \text { with } \quad \rho(\eta):=\int_{0}^{\eta} \partial_{2} f(r, r) d r=o(|\eta|) .
$$

For the proof we need the following comparison lemma (adapted from [35, pp. 64 and 72]):

3.9 LEMMA (COMPARISON LEMMA). Let the functions $\Psi, V, \delta_{1}, \delta_{2}, \rho_{1}, \rho_{2}$ be in $C^{0}([0, T], \mathbf{R}), \delta_{i} \geq 0, \Psi, V$ and $\rho_{i}$ differentiable on $\left.] 0, T\right]$. Assume that $g, \omega_{1}, \omega_{2}$ are real-valued functions with domains of definition $D(g), D\left(\omega_{i}\right) \subset \mathbf{R}^{2}$. Let $\boldsymbol{\Psi}, V$ be solutions of

$$
\left\{\begin{array}{l}
\Psi^{\prime}(t)=g(t, \Psi(t)) \\
-\delta_{1}(t) \leq V^{\prime}(t)-g(t, V(t)) \leq \delta_{2}(t)
\end{array} \quad \text { for } 0<t \leq T,\right.
$$

and assume that the functions $\rho_{i}$ satisfy the initial conditions

$$
\left\{\begin{array}{l}
0=\rho_{1}(0)=V(0)-\Psi(0)=\rho_{2}(0) \\
-\rho_{1}^{\prime}(0+)<0=V^{\prime}(0+)-\Psi^{\prime}(0+)<\rho_{2}^{\prime}(0+)
\end{array}\right.
$$

or

$$
-\rho_{1}(0)<V(0)-\Psi(0)<\rho_{2}(0)
$$

and the differential inequalities

$$
\rho_{i}^{\prime}(t)>\omega_{i}\left(t, \rho_{i}(t)\right)+\delta_{i}(t) \quad \text { for } 0<t \leq T
$$

the functions $\omega_{i}$ stem from unilateral estimates for the nonlinearity $g$ :

$$
\begin{cases}g(t, v)-g(t, v-\rho) \leq \omega(t, \rho) & \text { for all }(t, v-\rho) \in D(g) \\ g(t, v+\rho)-g(t, v) \leq \omega_{2}(t, \rho) & \text { for all }(t, v+\rho) \in D(g)\end{cases}
$$

Then we have

$$
\left.\left.-\rho_{1}(t)<V(t)-\Psi(t)<\rho_{2}(t) \quad \text { for all } t \in\right] 0, T\right] .
$$

PROOF OF 3.9. We only prove the second estimate of (3.24) (omitting the subscript 2); the other part is analogous. Because of (3.20) or (3.21), $V-\Psi<\rho$ for small $t>0$. Suppose $t_{0}>0$ is the first point with $V-\Psi=\rho$. Then we can estimate in $t_{0}$ :

$$
\begin{aligned}
V^{\prime}-\Psi^{\prime} & =V^{\prime}\left(t_{0}\right)-g\left(t_{0}, V\right)+g\left(t_{0}, V\right)-g\left(t_{0}, V-\rho\right) \\
& \leq \delta\left(t_{0}\right)+\left(t_{0}, \rho\right)<\rho^{\prime}\left(t_{0}\right)
\end{aligned}
$$

this is a contradiction.

PROOF OF 3.8. First of all, we apply Lemma 3.9, setting $g(\eta, \varsigma)=c-f_{0}(\eta) / \varsigma$ to prove

$$
\Psi(\eta) \leq V(\eta) \leq \Psi(\eta)+c \tau \rho(\eta) \text { for } 0<\eta \leq a .
$$

For this we have to verify the second line of (3.19) with suitable $\rho_{i}, \delta_{i}$ and $\omega_{i}$. As long as $V(\eta)>0$ we may use the graph equation (3.14) and the monotonicity properties of $u$ and $f$ to obtain

$$
V^{\prime}(\eta)-g(\eta, V)=\left(f_{0}(\eta)-f\left(\eta, \eta_{c \tau}\right)\right) / V \geq 0
$$


As long as $V^{\prime} \geq 0$, the mean value theorem gives (if $\eta=u(0)$ )

$$
f_{0}(\eta)-f\left(\eta, \eta_{c \tau}\right)=c \tau u(\xi) \partial_{2} f(\eta, u(\xi))<c \tau V(\eta) \partial_{2} f(\eta, \eta) \quad(-c \tau<\xi<c) .
$$

Moreover, for $\eta$ between 0 and $a$ and $\varsigma_{1}>\varsigma_{2}>0$ we have

$$
g\left(\eta, \varsigma_{1}\right)-g\left(\eta, \varsigma_{2}\right)=-f_{0}(\eta)\left(1 / \varsigma_{1}-1 / \varsigma_{2}\right) \leq 0 .
$$

So using $T=a, \delta_{1}=\omega_{1}=\omega_{2}=0, \delta_{2}(\eta)=c \tau \partial_{2} f(\eta, \eta)$ in Lemma 3.9, we obtain (3.24) for all $\rho_{1}, \rho_{2}$ with $\rho_{1}(0)=\rho_{2}(0)=0, \rho_{1}^{\prime}$ positive, $\rho_{2}^{\prime}>\delta_{2}$ on $\left.] 0, a\right]$, and that proves (3.25).

Now suppose that $V\left(\eta_{0}\right)=\Psi\left(\eta_{0}\right)=\varsigma_{0}>0$ for some $\left.\eta_{0} \in\right] 0,1[$, and let $u, \varphi$ be the corresponding solutions to $V, \Psi$ with $u(0)=\varphi(0)=\eta_{0}, u(0)=\varphi(0)=s_{0}$. Since $f$ is strictly quasimonotone we can verify $\operatorname{sign}\left(V^{\prime}-\Psi^{\prime}\right)\left(\eta_{0}\right)=\operatorname{sign}(u(0)-u(-c \tau))$. Since $u$ is strictly increasing on $\mathbf{R}^{-}$, we obtain $V^{\prime}\left(\eta_{0}\right)>\Psi^{\prime}\left(\eta_{0}\right)$ in contradiction to (3.25). The assumption $V\left(\eta_{1}\right)=\Psi\left(\eta_{1}\right)+c \tau \rho\left(\eta_{1}\right)$ for some $\eta_{1} \in[0,1[$ with $V^{\prime}\left(\eta_{1}\right) \geq 0$ leads to a contradiction in a similar way, and the estimate $\rho(\eta)=O(|\eta|)$ is obvious since $f$ is continuously differentiable and $\partial_{2} f(0,0)=0$.

We use this theorem to prove

3.10 TheOREM (CONTINUOUS Dependence of TrajeCtories). The unstable manifold of the FDE (3.3)-(3.4) depends continuously on $c$ in the following sense: Let $V_{c}$ be the solution of the graph equation (3.14)-(3.16) to the velocity $c$, let $\bar{c} \geq 0$ and $V_{\bar{c}}$ positive on $\left.] 0, \bar{\eta}\right]$. Then $V_{c}$ converges to $V_{\bar{c}}$ in $C^{1}([0, \bar{\eta}])$ for $c \rightarrow \bar{c}$.

ProOF. Let $\tau$ be positive, assume that $c \uparrow \bar{c}$ (the other case is analogous). For every $c>0$ let $u_{c}$ be the solution of $(3.2)$ with $u_{c}(0)=a, u_{c}(-\infty)=0$. On $[0, a]$ the equicontinuous monotonically increasing functions $V_{c}$ converge monotonically to a continuous function $V \leq V_{c}$. Lemma 3.6 shows convergence of the corresponding solutions $u_{c}$ to $u$ which can be shown to satisfy the FDE $(3.2)$ on ] $-\infty, 0$ ]. Everything else follows from the continuous dependence of solutions of FDEs [16, p. 41].

Before we show the existence of wave solutions, two lemmas discuss the possible behavior of solutions to (3.3)-(3.4) converging to the critical point $(1,0)$. with

3.11 LEMMA. If $V$ is a solution of the graph equation (3.14)-(3.16) on ]0,1[

$$
V(0)=V(1)=0, \quad V(\eta)>0 \quad \text { for } 0<\eta<1,
$$

then every corresponding solution $(u, v)$ of the FDE (3.3)-(3.4) satisfies

$$
0<u<1, \quad v>0 \quad \text { on } \mathbf{R} \text {. }
$$

PROOF. Because of Remark 3.2(ii) we may assume that $(u, v)$ satisfies the ODE (3.6) for $u(s) \in[0,1]$, so (3.26) immediately gives the weak inequality. Since 0,1 , and $u$ are stationary solutions of the parabolic equation (3.1), the strong maximum principle (Theorem 2.2) gives the result.

3.12 LEMMA. Assume that $u$ is a solution of the FDE (3.3) with

$$
1-\varepsilon<u<1, \quad u^{\prime} \text { positive on } \mathbf{R}^{+}, \quad u(\infty)=1 \text {. }
$$

If we define for $W \in L_{\mathrm{loc}}^{2}\left(\mathbf{R}^{+}\right)$

$$
\omega(w):=\inf \left\{\gamma \in \mathbf{R} \mid w e^{-\gamma} \in L^{2}\left(\mathbf{R}^{+}\right)\right\} \in \mathbf{R} \cup\{ \pm \infty\},
$$


then

(i) $\omega(u-1)=\omega(u) \in\{\mu(c),-\infty\}$,

(ii) $\lim _{s \rightarrow \infty} \dot{u}(s) /(u(s)-1)=\omega(u-1)$;

$\mu(c)$ is the negative eigenvalue of the linearization of (3.3) around $(1,0)$ (see (3.9)).

PROOF. Setting $y=u-1, y_{\tau}=y(\cdot-c \tau), g\left(y, y_{\tau}\right)=-f(u, u(\cdot-c \tau))$ and $g_{0}(y)=g(y, y)$, the FDE (3.3) can be written as

$$
y=c y+g\left(y, y_{\tau}\right)
$$

with the conditions

$$
-\varepsilon<y<0, \quad y>0 \text { and } \frac{g(y, z)}{y} \rightarrow \beta>0 \text { for } y, z \rightarrow \infty .
$$

(i) The equality can be proved by multiple partial integration using (3.30)-(3.31); since $y$ and $\dot{y}$ are bounded, you obtain

$$
\omega(y)=\omega(\dot{y})=\omega(\ddot{y})<0 ;
$$

the last inequality follows from [16, Chapter 10]. $y$ is a special solution of the linear equation

$$
\ddot{x}(s)-c \dot{x}(s)-a(s) x(s)-b(s) x(s-r)=0
$$

with $r=c \tau$ and the asymptotic behavior of the coefficients

$$
\left.\begin{array}{l}
a(s)=g_{0}(y(s)) / y(s) \rightarrow \beta>0 \\
b(s)=\left[g(y(s), y(s-r))-g_{0}(y(s))\right] / y(s-r) \rightarrow 0
\end{array}\right\} \quad \text { for } s \rightarrow \infty
$$

So we can apply Theorem 2 of [37] on the asymptotic behavior of linear FDEs with asymptotically constant coefficients and obtain

$$
\max _{\nu=0,1,2} \omega\left(y^{(\nu)}\right) \in \mathcal{M} \quad \text { with } \mathcal{M}=\left\{\operatorname{Re} \lambda \mid \lambda^{2}-c \lambda+\beta=0\right\} \cup\{-\infty\}
$$

together with (3.32) this completes the proof of (i).

To prove (ii) set $x:=\dot{y} / y ; x$ is negative, and we have to show $x(s) \rightarrow \omega(y)$ for $s \rightarrow \infty$. $x$ satisfies the equation

$$
\dot{x}(s)=c x-x^{2}+k(s) \quad \text { with } k(s)=\frac{g(y(s), y(s-r))}{y(s)} \rightarrow \beta \text { for } s \rightarrow \infty
$$

and one can show that $\mu(c)$ and $-\infty$ are the only possible limit points of $x(s)$ for $s \rightarrow \infty$. Since $\lim _{s \rightarrow \infty} y(s) / y(s)=a \in \mathbf{R}$ implies that for every $\varepsilon>0$ there are positive numbers $M_{\varepsilon}, S_{\varepsilon}$ such that

$$
M_{\varepsilon}^{-1} e^{(a-\varepsilon) s} \leq y(s) \leq M_{\varepsilon} e^{(a+\varepsilon) s} \quad \text { for } s \geq s_{\varepsilon}
$$

we obtain $x(s) \rightarrow \mu(c)$ iff $\omega(y)=\mu(c)$, and that proves (ii).

The next theorem contains the main result of this paragraph.

3.13 Theorem (Existence of WAVE SOlutions). Assume $\tau \geq 0$ and the condition on $f$

$$
\int_{0}^{1} f_{0}(r) d r \geq 0
$$




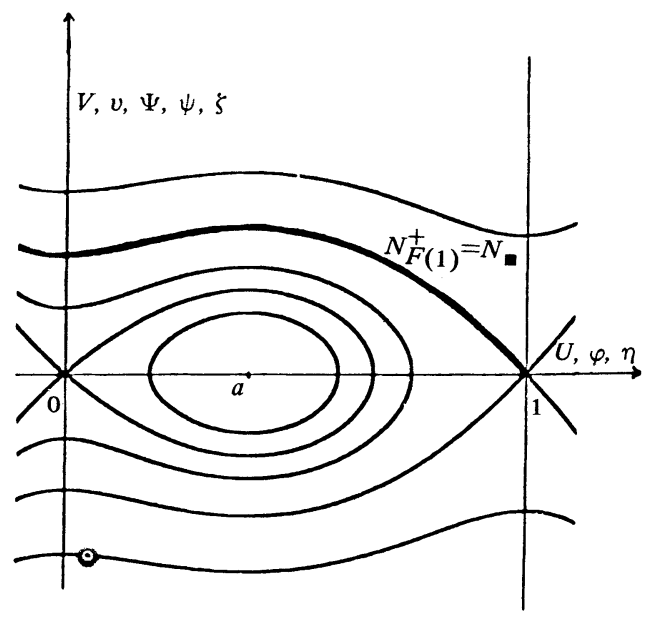

FIGURE 3.1

Then there is exactly one wave speed $c^{*} \geq 0$ such that (3.2) has a nontrivial strictly increasing wave solution with the asymptotic boundary values $u^{*}(-\infty)=0$, $u^{*}(\infty)=1$. Moreover $c^{*}=0$ iff the integral (3.34) vanishes.

3.14 REMARK. If (3.34) does not hold, one can transform the original equation (2.1) into $\partial_{t} W-\partial_{x}^{2} W=g(W, W(x, t-\tau))$ with $W=1-w, g(r, s)=-f(1-r, 1-s)$; $g$ satisfies conditions 3.1 and (3.34) so that (3.34) is no real restriction.

PROOF OF 3.13. If (3.34) vanishes there is a nontrivial wave solution of the ODE (3.5) with velocity 0 ; in this case, the ODE and FDE coincide, and Lemma 3.7(i) proves the second part of the theorem. Since the case $\tau=0$ is well known we can restrict ourselves to positive $\tau$ and $\int f_{0}>0$.

To investigate the behavior of the trajectories $V_{c}(\eta)$ we use the level sets (without the stationary points)

$$
N_{\kappa}:=\left\{(\eta, \zeta) \in \mathbf{R}^{2} \mid \frac{1}{2} \varsigma^{2}+F(\eta)=\kappa\right\} \backslash\{(0,0),(0, a),(0,1)\}, \quad \kappa \geq \kappa_{0} ;
$$

$F$ is the integral of $f_{0}$ with $F(0)=0, \kappa_{0}=F(a)<0$. The level sets $N_{*}$ are exactly the trajectories of solutions to (3.3) or (3.6) for $c=0$, i.e. every component of $N_{\kappa}$ corresponds (except for phase shift exactly) to one solution $\left(\varphi_{k}, \psi_{k}\right)$ of $(3.3)$ or (3.6); $\kappa \geq 0$ gives exactly the trajectories intersecting the $\zeta$-axis. For $\kappa \geq 0$ we define $N_{\kappa}^{+}:=N_{\kappa} \cap\{\varsigma>0\}$ and denote by $\Psi_{k}$ the solution of the graph equation (3.12) and (3.14) (for $c=0$ ) in $N_{\kappa}^{+}$; as usual, $V_{c}$ is the solution of the graph equation (3.14)-(3.16) for the velocity $c$. Now the proof is done in several steps:

Step 1. For positive $c, V_{c}$ intersects a level set $N_{\kappa}$ at most once, and in the point of intersection $\left(\eta_{k}, V\left(\eta_{k}\right)\right)=\left(\eta_{k}, \Psi_{k}\left(\eta_{k}\right)\right)$ holds

$$
V_{c}^{\prime}\left(\eta_{k}\right) \geq c+\Psi_{\kappa}^{\prime}\left(\eta_{\kappa}\right)
$$

"=" holds iff $\eta_{\kappa}=1$. 
ProOF OF 1. Let $(u, v)$ be a solution of (3.3)-(3.4) corresponding to $V_{c}$. Then we have

$$
\begin{aligned}
\frac{d}{d s}\left(\frac{1}{2} v^{2}(s)+F(u(s))\right) & =\frac{d}{d s} \kappa(s) \\
& =c v^{2}(s)+v(s) \int_{-c \tau}^{0} v(s+\mu) \partial_{2} f(u(s), u(s+\mu)) d \mu
\end{aligned}
$$

i.e. with increasing $s(u(s), v(s))$ "wanders" through "increasing" level sets. Estimate (3.35) can be verified directly.

The level set $N_{*}:=N_{F(1)}^{+}$through the critical point $(1,0)$ and the corresponding solution $\Psi_{*}:=\Psi_{F(1)}$ play a special role. We define

$$
\begin{aligned}
\underline{c}:=\inf \left\{c>0 \mid V_{c} \text { and } N_{*} \text { intersect in }\left(\eta_{c}, V_{c}\left(\eta_{c}\right)\right)\right. \\
\left.=\left(\eta_{c}, \Psi_{*}\left(\eta_{c}\right)\right) \text { with } 0<\eta_{c} \leq 1\right\} ;
\end{aligned}
$$

We will prove that this $\underline{c}$ is the desired wave speed $c^{*}$. Theorem 3.8 and the wellknown behavior of solutions to the ODE (3.6)-(3.7) ensure the $\underline{c}$ is finite.

Step 2. For every $c \geq \underline{c}$ there is a positive $\varepsilon$ such that $V_{c} \geq \varepsilon$ on $\left[\eta_{c}, \infty[\right.$.

PROOF OF 2. This statement follows directly from Step 1 and the shape of the level sets $N_{\kappa}^{+}$as shown in Figure 3.1.

Step 3. $\underline{c}$ is positive.

PROOF OF 3 . Step 2 shows that for $c \geq \underline{c} u_{c}(s)$ converges strictly monotonically to $+\infty$ for $s \rightarrow \infty$, and the continuous dependence of $u_{c}$ on $c$ ensures that $u_{c}$ is monotonically increasing on $\mathbf{R}$. Since $u_{0}(\infty)=0$ (see Figure 3.1) we obtain $\underline{c}>0$.

Step 4. $\eta_{c}$ converges monotonically from below to 1 for $c \downarrow \underline{c}$.

PROOF OF 4. The monotonic and continuous dependence of $\eta_{c}$ on $c$ is obvious (for $c \geq \underline{c}$ ). If $\eta_{\underline{c}}<1$, we obtain $V_{\underline{c}}^{\prime}\left(\eta_{\underline{c}}\right) \geq c+\Psi_{*}^{\prime}\left(\eta_{\underline{c}}\right)$ from Step 1 and the continuity Theorem 3.10. On the other hand we get $V_{\underline{c}}^{\prime}\left(\eta_{\underline{c}}\right)=\Psi_{*}^{\prime}\left(\eta_{\underline{c}}\right)$ or $\eta_{\underline{c}}=1$ from the definition (3.36) of $\underline{c}$; because of Steps 3 and 1 we obtain necessarily $\eta_{\underline{c}}=1$.

Step 5. $V_{\underline{c}}(1)=0$ and $V_{\underline{c}}^{\prime}(1)=\mu(c)$.

PROOF OF $5 . u_{c}$ is monotonically increasing on $\mathbf{R} ; u(\infty)=1$ follows since $V_{c}(1) \downarrow 0$ for $c \downarrow c$ and because of Lemma 3.5(iv). $V_{c}(1)=-\infty$ is impossible since $V_{c} \leq \Psi_{*}$, so Lemma 3.12 gives $V_{c}(1)=\mu(c)$.

Step 6. $\underline{c}$ is the unique wave speed $c^{*}$.

PROOF OF 6. We have to show that for every $c<\underline{c}$ there is an $\left.\eta_{c} \in\right] a, 1$ [ with $V_{c}\left(\eta_{c}\right)=0$. Suppose this is wrong; then there is a $c<\underline{c}$ with $\lim _{\eta \uparrow 1} V_{c}(\eta)=0$, and Lemma 3.12 gives $\lim _{\eta \uparrow 1} V_{c}^{\prime}(\eta) \in\{\mu(c),-\infty\}$. $V_{c}^{\prime}(\eta) \rightarrow-\infty$ gives a contradiction to $0<V_{c}<\Psi_{*}$ and $\Psi_{*}(1)=\mu(0)>-\infty$, so we must have $V_{c}^{\prime}(\eta) \rightarrow \mu(c)<0$ (see (3.9)). But this means that trajectories intersect in contradiction to Lemma 3.8, so Step 6 and the whole theorem are proved.

3.15 COROLlARY. The wave solution $u:=u^{*}$ from Theorem 3.13 shows the following asymptotic behavior: There are positive numbers $K_{1}, K_{2}$, and $\delta$ with

$$
\begin{gathered}
u^{(n)}(s)=K_{1} \lambda^{n}(c) \cdot e^{\lambda(c) s}\left(1+O\left(e^{\delta s}\right)\right) \quad \text { for } s \rightarrow-\infty, \\
(u-1)^{(n)}(s)=-K_{2} \mu^{(n)}(c) \cdot e^{\mu(c) s}\left(1+O\left(e^{-\delta s}\right)\right) \quad \text { for } s \rightarrow \infty,
\end{gathered}
$$

for $0 \leq n \leq 3$. 
ProOF. Because of (3.10) and Step 5 from the last proof there are positive constants $C_{1}, C_{2}$ with $C_{1} \leq u(s-c) / u(s) \leq C_{2}$ for all $s$. Since $f$ is in $C^{1, \nu}$ we may therefore apply Theorem 4.5 in [6, Chapter 13] on the asymptotic behavior of solutions to nonautonomous ODEs to obtain the results.

The last theorem of this section investigates the dependence of the wave speed and wave solution on the time lag:

3.16 THEOREM. (i) Let $\tau_{0} \geq 0$, and let $V_{\tau}$ and $\left(u_{\tau}, v_{\tau}\right)$ be the wave solution corresponding to $\tau \geq 0$ with $u_{\tau}(0)=a$. Then for $\tau \rightarrow \tau_{0} c(\tau)$ converges to $c\left(\tau_{0}\right), V_{\tau}$ uniformly on $[0,1]$ to $V_{\tau_{0}}$, and $\left(u_{\tau}, v_{\tau}\right)$ uniformly on $\mathbf{R}$ to $\left(u_{\tau_{0}}, v_{\tau_{0}}\right)$.

(ii) $c$ depends monotonically decreasing on $\tau$, especially

$$
0 \leq c(\tau) \leq c(0) \text { for all } \tau \geq 0 \text {. }
$$

Proof. (3.37) follows at once from Theorem 3.8 and the fact that $V_{c, \tau}^{\prime}(0)=$ $\lambda(c)$ increases strictly monotonically in $c$. The proof of the rest of the theorem is somewhat similar to the proof of Theorem 3.10 except that monotonic convergence can no longer be used.

(i) Because of (3.37) there is a sequence $\tau_{n} \rightarrow \tau_{0}$ such that $c_{n}:=c\left(\tau_{n}\right)$ converges to a $c_{0} \in[0, c(0)]$; let $V_{n}, u_{n}, v_{n}$ be the corresponding wave solutions to $c_{n}$. It is easy to see that the sets $A_{n}:=\left\{V_{n}^{\prime}(\eta) \mid \eta \geq 1\right\}$ are bounded for every $0 \leq \eta \leq a$, and so the Arzela-Ascoli theorem ensures uniform convergence (possibly of a subsequence) of $V_{n}$ to a continuous function $V_{0}$ on $[0, a]$. Now let $u_{n}$ be the wave solution corresponding to $V_{n}$ with $u_{n}(0)=a$, and define $u_{0}$ by

$$
u_{0}(a)=a, \quad u_{0}(s)=\eta \quad \text { with } s=\int_{a}^{\eta} \frac{d \sigma}{V_{0}(\sigma)} \rightarrow-\infty \text { for } \eta \rightarrow 0 .
$$

By integral representation of $u_{n}$ we can show that $u_{n} \rightarrow u_{0}$ uniformly on $\mathbf{R}, u_{0}$ satisfies the $\operatorname{FDE}(3.2)$ with $\tau_{0}$ and $c_{0}, u_{0}(\infty)=1$ and consequently $V_{n} \rightarrow V_{0}$ uniformly on $[0,1]$.

(ii) If we assume $\tau_{1}>\tau_{2}$ and $c\left(\tau_{1}\right)>c\left(\tau_{2}\right)$, we get a first intersection of the trajectories in some $\eta \in] 0,1\left[\right.$ with $V_{1}>V_{2}$ on ]0, $\eta[$. Using Lemma 3.6 and the graph equation (3.14) gives a contradiction to the necessary "intersection condition" $V_{1}^{\prime}(\eta) \leq V_{2}^{\prime}(\eta)$.

4. On the stability of traveling waves. In this paragraph we present a first step in the investigation of the stability of traveling waves constructed in the preceding paragraphs. These investigations only make sense in the transformed coordinate systems $s=x+c t$ in which traveling waves are stationary points. We are only interested in the linearized stability of wave solutions $\varphi_{\tau}$ of (2.1). The linearization of $(2.1)$ around $\varphi_{\tau}$ is

$$
\partial_{t} v(s, t)=\left(B^{\tau} v\right)(s, t)
$$

with

$$
\left(B^{\tau} v\right)(s, t)=\partial_{s}^{2} v-c \partial_{s} v+a_{\tau}(s) v(s, t)+b_{\tau}(s) v(s-c \tau, t-\tau)
$$

where the coefficients

$$
\left.\begin{array}{l}
a_{\tau}(s):=\partial_{1} f\left(\varphi_{\tau}(s), \varphi_{\tau}(s-c \tau)\right) \rightarrow a^{ \pm} \\
b_{\tau}(s):=\partial_{2} f\left(\varphi_{\tau}(s), \varphi_{\tau}(s-c \tau)\right) \rightarrow b^{ \pm}
\end{array}\right\} \quad \text { for } s \rightarrow \pm \infty
$$


approach their limits

$$
a^{-}:=\partial_{1} f(0,0), \quad a^{+}:=\partial_{1} f(1,1), \quad b^{-}:=\partial_{2} f(0,0), \quad b^{+}:=\partial_{2} f(1,1)
$$

exponentially fast (see $\S \S 2$ and 3 ). Let $U(t)$ be the semigroup determined by (4.1) on $B C^{0}:=B C^{0}(\mathbf{R} \times[-T, 0], C)$ with fixed $T>0$. The infinitesimal generator $A^{\tau}$ of $U(t)$ is given by the differential expression

$$
\left(L^{\tau} \psi\right)(s, \theta):= \begin{cases}\partial_{\theta} \psi(s, \theta) & \text { for } \theta<0 \\ \left(B^{\tau} \psi\right)(s, 0) & \text { for } \theta=0\end{cases}
$$

and the domain $D\left(A^{\tau}\right) \subset B C^{0}$ consisting of all sufficiently smooth functions with $L^{\tau} \psi \in B C^{0} ; D\left(A^{\tau}\right)$ depends genuinely on $\tau$.

In this section we derive estimates for the spectrum $\sigma\left(A^{\tau}\right)$ for small $\tau>0$ with the result. For wave solutions $\varphi_{\tau}$

-from $\S 2, \sigma\left(A^{\tau}\right)$ extends into the complex right half-plane $\mathbf{C}^{+}$

- from $\S 3, \sigma\left(A^{\tau}\right)$ lies completely in $\mathbf{C}^{-}$except for a simple isolated eigenvalue 0 coming from the translation invariance of (3.1) against $s \rightarrow s+s_{0}$.

If we consider equation (4.1) in suitably weighted spaces $B C_{w}^{0}:=$ $B C_{w}^{0}(\mathbf{R} \times[-T, 0], \mathbf{C})$, then the spectrum $\sigma\left(A_{w}^{\tau}\right)$ of the corresponding semigroup $U_{w}(t)$ (given (4.1), but operating on $B C_{w}^{0}$ ) lies in $\mathbf{C}^{-}$.

So at least for small $\tau>0, A^{\tau}$ has roughly the same spectral properties as in the well-known case without time delay. Although the results in this paragraph do not depend on the quasimonotonicity of $f$, I will only discuss the examples

$$
\begin{aligned}
& f\left(u, u_{\tau}\right)=u(1-u)\left(u_{\tau}-\alpha\right) \quad(\S 3, \text { "Huxley" nonlinearity }), \\
& f\left(u, u_{\tau}\right)=u_{\tau}(1-u) \quad(\S 2, \text { "KPP" nonlinearity }) .
\end{aligned}
$$

For (4.6) we will always assume $c=c^{*}$, for (4.7) $c>c^{*}>0$. In our discussion we have to treat the essential spectrum and the eigenvalues separately. We use the definition of essential spectrum given in [14] and denote the resolvent set, set of normal points, and essential spectrum of an operator $L$ by $\rho(L), \tilde{\rho}(L)$, and $\sigma_{m}(L)$ respectively.

The following two lemmas are easy to prove and establish a connection between the spectrum of $A^{\tau}$ and the spectral properties of simpler operators:

4.1 LEMma. Let $T_{\lambda}^{\tau}: D\left(T_{\lambda}^{\tau}\right)=B C^{2}(\mathbf{R}, \mathbf{C}) \subset B C^{0}(\mathbf{R}, \mathbf{C}) \rightarrow B C^{0}(\mathbf{R}, \mathbf{C})$ be given by

$$
T_{\lambda}^{\tau} u(s):=u^{\prime \prime}(s)-c u^{\prime}(s)+\left(a_{\tau}(s)-\lambda\right) u(s)+e^{-\lambda \tau} b_{\tau}(s) u(s-c \tau) .
$$

Then $\lambda$ is a regular point (normal point, in the essential spectrum) of $A^{\tau}$ iff 0 is a regular point (normal point, in the essential spectrum) of $T_{\lambda}^{\tau}$.

4.2 LEMMA. Let $M_{\lambda}^{\tau}: D\left(M_{\lambda}^{\tau}\right)=B C^{2}(\mathbf{R}, \mathbf{C}) \times B C^{1}(\mathbf{R}, \mathbf{C}) \subset B C^{0}\left(\mathbf{R}, \mathbf{C}^{2}\right) \rightarrow$ $B C^{0}\left(\mathbf{R}, \mathbf{C}^{2}\right)$ be given by

$$
M_{\lambda}^{\tau}\left(\begin{array}{l}
u \\
v
\end{array}\right)=\left(\begin{array}{l}
u^{\prime}-v \\
v^{\prime}-c v+\left(a_{\tau}-\lambda\right) u+b_{\tau} e^{-\lambda \tau} u(\cdot-c \tau)
\end{array}\right) .
$$

Then 0 has the same spectral properties for $T_{\lambda}^{\tau}$ and $M_{\lambda}^{\tau}$. Lemma 4.1 is a motivation for the following definitio.. . 
4.3 Definition. Let $X$ be a Banach space of functions $u: \mathbf{R} \rightarrow \mathbf{C}^{n}$. For an operator $B^{\tau}: D\left(B^{\tau}\right) \subset X \rightarrow X$ of the form $B^{\tau}=B_{1}^{\tau}+B_{2}^{\tau}$ with

$$
\begin{aligned}
& B_{1}^{\tau} u(s)=a_{2 \tau}(s) u^{\prime \prime}(s)+a_{1 \tau}(s) u^{\prime}(s)+a_{0 \tau}(s) u(s), \\
& B_{2}^{\tau} u(s)=b_{\tau}(s) u\left(s-c_{\tau} \tau\right), \quad c_{\tau}>0,
\end{aligned}
$$

with bounded coefficients from $\mathbf{R}^{(n, n)}$ which do not all vanish, define the operator

$$
B_{\lambda}^{\tau}:=B_{1}^{\tau}-\lambda \operatorname{Id}+e^{-\lambda \tau} B_{2}^{\tau}
$$

and the operator-valued function

$$
R\left(\lambda, B^{\tau}\right)=\left(B_{\lambda}^{\tau}\right)^{-1} .
$$

REMARKS ON THE NOTATION. In cases where no misunderstanding is possible, I will often skip the index $\tau$ and the arguments $s$ and $\theta$ and especially write $c$ or $c_{\tau}$ for $c(\tau), c_{0}$ for $c(0)$. For abbreviation, we will consistently use the following notation: The dependence of the time lag will be denoted by a superscript $\tau$ or 0 with operators and functions, and by a subscript with (possibly variable) coefficients. In the case of functions, a subscript 0 denotes the restriction on the line $\theta=0$. Furthermore we will write $R_{\lambda}$ for $R(\lambda, B)$. For $\left.\tau=0, R_{\lambda}^{0}=\left(B^{0}-\lambda\right)^{-1}\right)$ is the normal resolvent (which will always be denoted by $(B-\lambda)^{-1}$ ).

4.4 LEMMA. $R_{\lambda}$ is an analytic function of $\lambda$ and satisfies

$$
R_{\lambda}-R_{\mu}=(\lambda-\mu)\left(R_{\lambda} R_{\mu}-\frac{e^{-\lambda \tau}-e^{-\mu \tau}}{\lambda-\mu} R_{\lambda} B_{2}^{\tau} R_{\mu}\right) .
$$

If $T^{\tau}=T_{1}^{\tau}+T_{2}^{\tau}$ with $T_{1}^{\tau} u=u^{\prime \prime}-c u^{\prime}+a_{\tau} u, T_{2}^{\tau} u=b_{\tau} u(\cdot-c \tau)$, then (see Lemma 4.1) $\rho\left(A^{\tau}\right)$ is the region of holomorphy of $R\left(\cdot, T^{\tau}\right)$, and $\tilde{\rho}\left(A^{\tau}\right)$ is the region of meromorphy of $R\left(\cdot, T^{\tau}\right)$.

We can now prove a theorem bounding the essential spectrum of $A^{\tau}$; the proof roughly follows Henry's book [17, pp. 138-141].

4.5 Theorem. Suppose $X=B C^{0}(\mathbf{R} \times[-T, 0], \mathbf{C}), A^{\tau}: D\left(A^{\tau}\right) \subset X \rightarrow X$ is given by $(4.5)$, (4.2) with coefficients $a_{\tau}(s), b_{\tau}(s)$ converging to $a_{ \pm}, b_{ \pm}$for $s \rightarrow+\infty$. Let

$$
S_{ \pm}^{\tau}:=\left\{\lambda \in \mathbf{C} \mid-y^{2}-i c y+a_{ \pm}+b_{ \pm} e^{-\lambda \tau} e^{i c y \tau}=0 \text { for } a y \in \mathbf{R}\right\}
$$

Then $S_{+}^{\tau}$ and $S_{-}^{\tau}$ each consist of a curve which is symmetric about the real axis and asymptotically parabolic, i.e. $\lambda=-y^{2}+O(|y|)$ for $|y| \rightarrow \infty$. Let $P$ denote the union of the regions inside or on the curves $S_{+}^{\tau}, S_{-}^{\tau}$; thus $\mathbf{C} \backslash P$ is the component of $\mathrm{C} \backslash\left(S_{+}^{\tau} \cup S_{-}^{\tau}\right)$ containing a right half plane. Then the essential spectrum of $A^{\tau}$ is contained in $P$, and in particular includes $S_{+}^{\tau} \cup S_{-}^{\tau}$.

The meaning of the curves $S_{ \pm}^{\tau}$ will be more obvious in the proof, and the significance for the examples (4.6)-(4.7) will be demonstrated in 4.9.

For the proof of the theorem, we have to analyze the region of meromorphy of $R\left(\lambda, T^{\tau}\right)$ according to Lemmas 4.1 and 4.4.

The following proposition reduces this problem to the solvability of a simpler equation. 
4.6 Proposition. Suppose $X$ is a Banach space of functions $u: \mathbf{R} \rightarrow \mathbf{C}$, $T: D(T) \subset X \rightarrow X$ a closed linear operator, $B: D(B) \subset X \rightarrow X$ a closed linear operator with $D(T) \subset D(B)$, and $B$ relatively compact with respect to $T$.

Suppose further that $B$ and $T$ can be decomposed as in Definition 4.4 as $T=$ $T_{1}+T_{2}, B=B_{1}+B_{2}$ (i.e. the shift terms are separated as $T_{2}$ and $\left.B_{2}\right)$. Let $G$ be a region in $\mathbf{C}$ where $R(\cdot, T)$ is holomorphic. Then we have: If $R\left(\lambda_{0}, T+B\right)$ is defined for one $\lambda_{0} \in G$, then $R(\cdot, T+B)$ is meromorphic on all $G$.

Since this proposition and its proof are only a slight modification of $[\mathbf{1 4}$, p. 22] we are not going to prove it. The proposition is used to simplify the original problem arising from the variable coefficients in the following way: Let $T^{*}$ be the extension of $T^{\tau}$ to the maximal domain $D\left(T^{*}\right) \subset B C_{*}^{0}(\mathbf{R}, \mathbf{C})$ where $B C_{*}^{k}\left(\mathbf{R}, \mathbf{C}^{n}\right)$ denotes the space of $\mathbf{C}^{n}$-valued functions $u$ that coincide with a $u^{+} \in B C^{k}\left(\mathbf{R}_{0}^{+}, \mathbf{C}^{n}\right)$ on $\mathbf{R}^{+}$and with a $u^{-} \in B C^{k}\left(\mathbf{R}_{0}^{-}, \mathbf{C}^{n}\right)$ on $\mathbf{R}^{-}$. Because of $\sigma_{\text {ess }}\left(T^{\tau}\right) \subset \sigma_{\text {ess }}\left(T^{*}\right)$ it is sufficient to give a bound for $\sigma_{\text {ess }}\left(T^{*}\right)$. Now define $\tilde{T}$ by $D(\tilde{T})=D\left(T^{*}\right)$ and $\tilde{T} u:=u^{\prime \prime}-c u^{\prime}+\tilde{a} u+\tilde{b} u(\cdot-c \tau)$ with coefficients $\tilde{a}(s), \tilde{b}(s)=a_{ \pm}, b_{ \pm}$for $\pm s>0$.

Since $\tilde{T}-T^{*}$ is relatively compact with respect to $\tilde{T}$ and $T^{*}$ in $B C_{*}^{0}$, Proposition 4.7 says that it suffices to study the solvability in $B C_{*}^{0}$ of the FDE

$$
\tilde{T}_{\lambda} u:=u^{\prime \prime}-c u^{\prime}+(\tilde{a}-\lambda) u+\tilde{b} u(\cdot-c \tau) .
$$

To prove Theorem 4.5 we consider the system

$$
u^{\prime}=v, \quad v^{\prime}=c v-(\tilde{a}-\lambda) u-\tilde{b} e^{-\lambda \tau} u(\cdot-c \tau)
$$

equivalent to $\tilde{T}_{\lambda} u=0$, or with $z=(u, v)^{t}, z_{s} \in C=C^{0}\left([-c \tau, 0], \mathbf{C}^{2}\right)$,

$$
z^{\prime}(s)=L_{\lambda}(s) z_{s}
$$

with

$$
L_{\lambda}(s):= \begin{cases}L_{\lambda}^{+} & \text {for } s>0 \\ L_{\lambda}^{-} & \text {for } s<0\end{cases}
$$

If we set

$$
\left(\tilde{M}_{\lambda} z\right)(s):=z^{\prime}(s)-L_{\lambda}(s) z_{s}
$$

then studying the solutions to (4.16) in $B C_{*}^{0}\left(\mathbf{R}, \mathbf{C}^{2}\right)$ is equivalent to studying the solvability in $\tilde{M}_{\lambda} z=0$ in $B C_{*}^{0}\left(\mathbf{R}, \mathbf{C}^{2}\right)$ or of $\tilde{T}_{\lambda} u=0$ in $B C_{*}^{0}(\mathbf{R}, \mathbf{C})$.

$S^{\tau}$ is the set of $\lambda \in \mathbf{C}$ for which the infinitesimal generator $A_{\lambda}^{ \pm}$of $L_{\lambda}^{ \pm}$(as a mapping from $C$ to $C$ ) has an imaginary eigenvalue. Since $L_{\lambda}^{+}$and $L_{\lambda}^{-}$depend analytically on $\lambda$, Theorem 4.5 follows immediately from the following

4.7 LEMMA. Suppose the linear mappings $L_{\lambda}^{+}, L_{\lambda}^{-}: C \rightarrow \mathbf{C}^{2}$ depend analytically on $\lambda \in \mathbf{C}$ and that $A_{\lambda}^{ \pm}$is the infinitesimal generator to $L_{\lambda}^{ \pm}$. Let

$$
S_{ \pm}:=\left\{\lambda \in \mathbf{C} \mid A_{\lambda}^{ \pm} \text {has an imaginary eigenvalue }\right\} .
$$

Let $\tilde{M}_{\lambda}$ be given by (4.17) as a closed, densely defined operator in $B C_{*}^{0}\left(\mathbf{R}, \mathbf{C}^{2}\right)$ with $D\left(\tilde{M}_{\lambda}\right)=B C_{*}^{2}(\mathbf{R}, \mathbf{C}) \times B C_{*}^{1}(\mathbf{R}, \mathbf{C})$. Then if $G$ is any open connected set in $\mathbf{C} \backslash\left(S_{+} \cup S_{-}\right)$, either

(i) $0 \in \sigma\left(\tilde{M}_{\lambda}\right)$ for all $\lambda \in G$, or

(ii) $0 \in \tilde{\rho}\left(\tilde{M}_{\lambda}\right)$ for all $\lambda \in G$.

Also, $0 \in \sigma_{\text {ess }}\left(\tilde{M}_{\lambda}\right)$ whenever $\lambda \in S_{+} \cup S_{-}$. 
This lemma completes the proof of Theorem 4.6: If $G \subset \mathbf{C} \backslash\left(S_{+} \cup S_{-}\right)$contains a right half plane, then $G \subset \tilde{\rho}\left(T^{*}\right)$ since sufficiently large $\lambda \in \mathbf{R}^{+}$are regular values of $T^{*}$.

To prove Lemma 4.7 we need a criterion when 0 is a regular point of $\tilde{M}_{\lambda}$. This is given by the following

4.8 LEMMA. Let 0 be a hyperbolic critical point of $L^{+}$and $L^{-}$(i.e. there are no imaginary eigenvalues of $A^{+}$and $\left.A^{-}\right), C:=C^{0}\left([-c \tau, 0], \mathbf{C}^{2}\right), P^{ \pm}: C \rightarrow C$ projections to the eigenvalues of $A^{ \pm}$in $\mathrm{C}^{+}\left(P^{ \pm}\right.$are finite-dimensional according to $\left[16\right.$, Chapter 7]), $Q^{ \pm}:=\mathrm{Id}-P^{ \pm}$the projections to the "stable" generalized eigenspaces of $A^{ \pm}$. Let $L(s)=L^{+}$for $s>0, L(s)=L^{-}$for $s<0$. Then the equation

$$
\tilde{M} z(s)=z^{\prime}(s)-L(s) z_{s}=f(s), \quad s \in \mathbf{R},
$$

is uniquely solvable for arbitrary $f \in B:=B C_{*}^{0}\left(\mathbf{R}, \mathbf{C}^{2}\right)$ iff $C$ can be decomposed as

$$
C=R\left(Q^{+}\right) \oplus R\left(P^{-}\right) \text {. }
$$

In this case the solution operator $K: B \rightarrow B, K(f)=z$ is continuous.

ProOF OF 4.8. We have $C=R\left(Q^{+}\right)+R\left(P^{-}\right)$iff

$$
R\left(P^{+}\right)+R\left(Q^{-}\right) \subset R\left(Q^{-}\right)+R\left(P^{-}\right)
$$

and (4.19) is a direct composition iff the "=" sign holds in (4.20). In this proof, let the superscript $*$ stand for + or - . According to [16, Theorem 9.1.1], (4.18) restricted to $\mathbf{R}^{*}$ is solvable for all $f \in B^{*}:=B C^{0}\left(\mathbf{R}^{*}, \mathbf{C}^{2}\right)$ iff 0 is a hyperbolic critical point of $L^{*}$, and a special solution bounded on $\mathbf{R}^{*}$ is given by the operators $K^{*}: B^{*} \rightarrow B^{*}$

$$
\begin{aligned}
& \left(K^{+} f\right)_{s}=\int_{0}^{s} T^{+}(s-h)\left(Q^{+} X_{0}\right) f(h) d h-\int_{s}^{\infty} T^{+}(s-h)\left(P^{+} X_{0}\right) f(h) d h \\
& \left(K^{-} f\right)_{s}=\int_{-\infty}^{s} T^{-}(s-h)\left(Q^{-} X_{0}\right) f(h) d h-\int_{s}^{0} T^{-}(s-h)\left(P^{-} X_{0}\right) f(h) d h
\end{aligned}
$$

here $s \in \mathbf{R}^{*}, T^{*}(t)$ denotes the semigroup generated by $A^{*}, X_{0}(\theta)=0$ for $-c \tau \leq$ $\theta<0, X_{0}(0)=\left(\begin{array}{ll}1 & 0 \\ 0 & 1\end{array}\right)$, and $\left(K^{*} f\right)_{s}=\left.\left(K^{*} f\right)\right|_{[s-c \tau, s]} \in C$ (cf. [16, §9.1 and $\left.\left.\S 7.6\right]\right)$. The general solution $z^{*}(\cdot, \phi, f) \in B^{*}$ of (4.18) on $\mathbf{R}^{*}$ with initial or final value $z_{0}^{*}=\phi \in C$ has the form

$$
\begin{array}{ll}
z_{s}^{+}=T^{+}(s)\left(Q^{+} \phi\right)+\left(K^{+} f\right)_{s}, & s \geq 0, \\
z_{s}^{-}=T^{-}(s)\left(P^{-} \phi\right)+\left(K^{-} f\right)_{s}, & s \leq 0 .
\end{array}
$$

The special case $s=0$ shows at once

$$
P^{+} \phi=\left(K^{+} f\right)_{0}, \quad Q^{-} \phi=\left(K^{-} f\right)_{0},
$$

i.e. the boundedness of $z^{*}(\cdot, \phi, f)$ on $\mathbf{R}^{*}$ forces the relations

$$
\begin{aligned}
& P^{+} \phi=-\int_{0}^{\infty} T^{+}(-h)\left(P^{+} X_{0}\right) f(h) d h, \\
& Q^{-} \phi=\int_{-\infty}^{0} T^{-}(-h)\left(Q^{-} X_{0}\right) f(h) d h .
\end{aligned}
$$


Let $z=z(\phi) \in C^{0}(\mathbf{R})$ be the "combined" solution of (4.18) on $\mathbf{R}$ with $z_{\mid \mathbf{R}^{*}}=z^{*}$, and especially $z_{0}=\phi \cdot z$ is bounded on $\mathbf{R}^{+}$iff $P^{+} z_{0} \in R\left(Q^{+}\right)+R\left(P^{-}\right)$, and bounded on $\mathbf{R}^{-}$iff $Q^{-} z_{0} \in R\left(P^{-}\right)+R\left(Q^{+}\right)$, so (4.20) is sufficient (and necessary, as is easily seen) for the solvability of (4.18) on $\mathbf{R}$. Furthermore uniqueness holds iff $R\left(Q^{+}\right) \cap R\left(P^{-}\right)=\{0\}$, and the estimates used to show uniqueness also yield the desired estimate

$$
\left\|z_{s}\right\|_{C} \leq \mathrm{const}\|f\|_{L^{\infty}(\mathbf{R})} \quad \text { for all } s \in \mathbf{R} .
$$

ProOF OF 4.7. The projections $Q_{\lambda}^{ \pm}, P_{\lambda}^{ \pm}$to $L_{\lambda}^{ \pm}$(defined as in Lemma 4.8) depend analytically on $\lambda$ in $G$. Analogously to [16, p. 173 and $\S 7.3]$ we can define a bilinear form $(\cdot, \cdot)_{\lambda}: C \times C \rightarrow \mathbf{C}$ to $L_{\lambda}^{ \pm}$such that $P_{\lambda}^{ \pm}, Q_{\lambda}^{ \pm}$are orthogonal with respect to $(\cdot, \cdot)_{\lambda} ;(\cdot, \cdot)_{\lambda}$ also depends analytically on $\lambda$. The condition $C=R\left(Q_{\lambda}^{+}\right) \oplus R\left(P_{\lambda}^{-}\right)$ (necessary and sufficient for $0 \in \rho\left(\tilde{M}_{\lambda}\right)$ according to Lemma 4.9) is equivalent to

$$
\operatorname{dim} R\left(P_{\lambda}^{+}\right)=\operatorname{dim} R\left(P_{\lambda}^{-}\right)=: n(\lambda)
$$

and

$$
\psi(\lambda):=\operatorname{det}\left(\left(\left(e_{i}^{-}(\lambda), e_{j}^{+}(\lambda)\right)_{\lambda}\right)_{1 \leq i, j \leq n(\lambda)}\right) \neq 0
$$

$e_{i}^{ \pm}(\lambda)$ are basis vectors of $P_{\lambda}^{ \pm}(C)$ (cf. [16, Lemma 7.33] or the introduction to Proposition 2.6). Because of $G \subset \mathbf{C} \backslash\left(S_{+} \cup S_{-}\right), n(\lambda)$ is constant on $G$, and since $\psi$ is analytic we have either $\psi \equiv 0$ on $G$, or $\psi(\lambda) \neq 0$ for all $\lambda \in G$ except for isolated zeroes of finite multiplicity corresponding to finite poles of $\left(\tilde{M}_{\lambda}\right)^{-1}$; that shows the alternative (i) or (ii). The remaining part of the proof can be copied directly from Henry [17, p. 139].

4.9 EXAMPLES FOR THEOREM 4.5. To show the significance of Theorem 4.5 we have to analyze the shape of the sets $S_{ \pm}^{\tau}$. Omitting the subscript \pm we have

$$
S^{\tau}=\{\mu+i \nu \in \mathbf{C} \mid F(\mu, \nu, \tau, y)=0 \text { for a } y \in \mathbf{R}\}
$$

with $F: \mathbf{R}^{4} \rightarrow \mathbf{C}$ given by

$$
F(\mu, \nu, \tau, y)=-y^{2}+i c y+b e^{-\tau(c y+\nu)} e^{-\tau \mu}-\mu-i \nu .
$$

For $\tau=0$, the set

$$
S^{0}=\left\{-\nu^{2} / c^{2}+a+b+i \nu \mid \nu \in \mathbf{R}\right\}
$$

is parametrizable over $\nu \in \mathbf{R}$. For the Huxley nonlinearity

$$
f\left(u, u_{\tau}\right)=u(1-u)\left(u_{\tau}-\alpha\right)
$$

we have $a_{+}=\alpha-1, a_{-}=-\alpha, b_{+}=b_{-}=0$. So $S^{\tau}$ does not depend on $\tau$, and for all $\tau>0$ it holds that

$$
\sigma_{\text {ess }}\left(A^{\tau}\right) \subset\{\lambda \in \mathbf{C} \mid \operatorname{Re} \lambda \leq \max (\alpha-1,-\alpha)<0\} .
$$

For the KPP nonlinearity

$$
f\left(u, u_{\tau}\right)=u_{\tau}(1-u)
$$

we have $a_{+}=-1, a_{-}=b_{+}=0, b_{-}=1$. So $\lambda=1$ is in the essential spectrum of $A^{0}$, and by the implicit function theorem this instability is conserved for small $\tau>0$. 
Introducing weighted norms (according to Sattinger's ideal $[\mathbf{2 8}, \mathbf{2 9}]$ ) changes the stability properties. It is easy to carry over Henry's presentation $[\mathbf{1 7}$, p. 141] to our situation to see that the essential spectrum can be shifted into the left half plane in the case of the KPP nonlinearity, but at the same time 0 ceases to be an eigenvalue since the eigenfunction $\varphi^{\prime}$ of $T^{\tau}$ ( $\varphi$ is the traveling wave solution from $\S 2$ ) is no longer bounded in the weighted norm.

We still have to analyze the eigenvalues of the infinitesimal generator $A^{\tau}$. In the following we will always assume that the essential spectrum of $A^{\tau}$ lies strictly in the complex left half plane, possibly by working in suitable weighted spaces. If this is the case and therefore zero is a regular value of $A^{\tau}$, the argument is correspondingly simpler.

At first we show that the operators $A^{\tau}$ depend continuously on $\tau$ in a certain sense. Since the domains $D\left(A^{\tau}\right)$ genuinely depend on $\tau$ we will show that $A^{\tau}$ converges to $A^{0}$ in the generalized sense (following Kato [22, p. $\left.197 \mathrm{ff}\right]$ ). The following notations are essential for the following:

4.10 Definition. Let $Z$ be a Banach space, $M$ and $N$ (nontrivial) closed subspaces of $Z$. We set

$$
\begin{aligned}
& \delta(M, N):=\inf \{\delta>0 \mid \operatorname{dist}(u N) \leq \delta\|u\| \text { for all } u \in M\}, \\
& \hat{\delta}(M, N):=\max (\delta(M, N), \delta(N, M)) .
\end{aligned}
$$

$\hat{\delta}$ is called the $g a p$ between $M$ and $N$. For closed operators $A, B$ on a Banach space $X$ the gaphs $G(A), G(B)$ are closed subspaces $X \times X$, and the gap between $A$ and $B \hat{\delta}(A, B)$ is defined by

$$
\delta(A, B)=\delta(G(A), G(B)), \quad \hat{\delta}(A, B)=\hat{\delta}(G(A), G(B)) .
$$

$A_{n}$ converges to $A$ in the generalized sense (for short: $A_{n} \rightarrow A$ ) iff $\hat{\delta}\left(A, A_{n}\right) \rightarrow 0$ for $n \rightarrow \infty$.

Since the spectrum in general is only upper half continuous with respect to generalized convergence, Theorem 4.6 and its consequences cannot be derived by the following method. Since, however, simple eigenvalues depend continuously on $A^{\tau}$, we will first prove the following proposition used in later proofs:

4.11 Proposition. Assume that for $\tau \geq 0 A^{\tau}$ is the infinitesimal generator of the semigroups $U^{\tau}(t)$ given by (4.1) operating on $B C^{0}$. Then for small $\tau>0$, zero is either a regular value or an isolated algebraically simple eigenvalue of $A^{\tau}$; zero is an eigenvalue of $A^{\tau}$ iff it is an eigenvalue of $A^{0}$.

ProOF. For $\tau=0$ the situation is known (e.g. [28]). According to Lemma 4.2 we have to study the bounded solutions of

$$
T_{0}^{\tau} u=u^{\prime \prime}-c_{\tau} u^{\prime}+a_{\tau} u+b_{\tau} u(\cdot-c \tau)=0 .
$$

The relative bound $[\mathbf{2 2}$, p. 190] of the operator

$$
\left(T_{0}^{\tau}-T_{0}^{0}\right) u=\left(c_{0}-c_{\tau}\right) u^{\prime}+\left(a_{\tau}-a_{0}\right) u+b_{\tau}(u(\cdot-c \tau)-u)+\left(b_{\tau}-b_{0}\right) u
$$

with respect to $T_{0}^{0}$ vanishes for $\tau \rightarrow 0$, so $T_{0}^{\tau} \rightarrow T_{0}^{0}$ [22, Theorem IV-2.14, p. 203], and the stability theorem $[\mathbf{2 2}, \mathrm{IV}-\S 3.5$, p. 213] gives the desired result.

The next theorem shows that the infinitesimal generators depend continuously on $\tau$ in the sense of Definition 4.11: 
4.12 THEOREM. Let $A^{\tau}, \tau \geq 0$, be the infinitesimal generator of the semigroup $U^{\tau}(t)$ given by (4.1-5) and operating on $B C^{0}$. Then $A^{\tau} \rightarrow A^{0}$ for $\tau \rightarrow 0$.

PROOF. Since the usual criteria for generalized convergence do not apply in our case, we have to go back to the definition. First we show that for all $\tau \rightarrow 0$ there is $z^{0}(\tau) \in G\left(A^{0}\right)$ and $z^{\tau} \in G\left(A^{\tau}\right)$ such that

$$
\left\|z^{0}(\tau)\right\|=1 \quad \text { and } \quad\left\|z^{0}(\tau)-z^{\tau}\right\| \rightarrow 0 \quad \text { for } \tau \rightarrow 0
$$

If this holds, then we have at once $\lim \sup _{\tau \rightarrow 0} \hat{\delta}\left(A^{0}, A^{\tau}\right)=0$, and the theorem is proved.

So let $z^{0}=\left(u^{0}, v^{0}\right), z^{\tau}=\left(u^{\tau}, v^{\tau}\right)$ with $v^{0}=A^{0} u^{0}, v^{\tau}=A^{\tau} v^{\tau}$, and let $\left\|z^{0}\right\|=$ $\left\|u^{0}\right\|+\left\|v^{0}\right\|=1$. Then

$$
v^{0}=A^{0} u^{0}= \begin{cases}\partial_{\theta} u^{0} & \text { for } \theta<0 \\ u_{0}^{0^{\prime \prime}}-c_{0} u_{0}^{0^{\prime}}+\left(a_{0}+b_{0}\right) u_{0}^{0} & \text { for } \theta=0\end{cases}
$$

To satisfy (4.31) we set

$$
u^{\tau}(s, \theta)=u^{0}(s, \theta)+h_{\tau}(s)
$$

and obtain

$$
A^{\tau} u^{\tau}-A^{0} u^{0}= \begin{cases}0 & \text { for } \theta<0 \\ H^{\tau} h_{\tau}+S^{\tau} u^{0} & \text { for } \theta=0\end{cases}
$$

with

$$
H^{\tau} w=w^{\prime \prime}-c_{\tau} w^{\prime}+a_{\tau} w+b_{\tau} w\left(\cdot-c_{\tau} \tau\right)
$$

and

$$
S^{\tau} u^{0}=\left(c_{0}-c_{\tau}\right) u_{0}^{0^{\prime}}+\left(a_{\tau}-a_{0}\right) u_{0}^{0}+b_{\tau} u^{0}\left(\cdot-c_{\tau} \tau,-\tau\right)-b_{0} u_{0}^{0} .
$$

It is easy to verify $\left\|S^{\tau} u^{0}\right\| \rightarrow 0$ for $\tau \rightarrow 0$. So if we manage to find $h_{\tau}$ such that

$$
H^{\tau} h_{\tau}=-S^{\tau} u^{0}
$$

and

$$
\left\|h_{\tau}\right\| \rightarrow 0 \text { for } \tau \rightarrow 0
$$

then we have

$$
\left\|u^{\tau}-u^{0}\right\|+\left\|A^{\tau} u^{\tau}-A^{0} u^{0}\right\|=\left\|h_{\tau}\right\| \rightarrow 0 \text { for } \tau \rightarrow 0,
$$

i.e. (4.31) holds and the proof is finished.

To find $h_{\tau}$ we first note that 0 is an isolated algebraically simple eigenvalue of $H^{\tau}$ with eigenspace $N^{\tau}=\left\langle\varphi_{\tau}^{\prime}\right\rangle$. (The proof is a simplification of Proof 4.11.) Since $H^{\tau}$ and the projection Id $-\pi_{\tau}$ of $B C^{0}(\mathbf{R}, \mathbf{C})$ onto $N^{\tau}$ depend continuously on $\tau$, $H^{\tau} \circ \pi_{\tau}$ is bounded invertible, and one can show [22, p. 196] that the norm of $H^{\tau} \circ \pi_{\tau}$ is uniformly bounded for small $\tau \geq 0$. So $h_{\tau}:=-\left(H^{\tau} \circ \pi_{\tau}\right)^{-1}\left(S^{\tau} u^{0}\right)$ satisfies all requirements, and the proof is complete.

So finally we can show that for small $\tau \geq 0$ the spectrum of the infinitesimal generators $A^{\tau}$ has similar "stability properties" as for $\tau=0$. 
4.13 THEOREM. There are positive numbers $\delta$ and $\tau_{0}$ such that the following holds: Let $U^{\tau}(t)$ be the semigroup given by (4.1) operating on $B C^{0}$ (possibly with a suitable weighted norm). Then for $0 \leq \tau \leq \tau_{0}$ the spectrum of the infinitesimal generator $A^{\tau}$ of $U^{\tau}(t)$ lies (possibly except for a simple isolated eigenvalue zero) in the region $\{\lambda \in \mathbf{C} \mid \operatorname{Re} \lambda \leq-\delta\}$.

PROOF. For $\tau=0$ the spectrum even satisfies a cone condition: There are $\delta_{0}$, $\varepsilon>0$ such that

$$
\sigma\left(A^{0}\right) \subset\left\{\lambda \in \mathbf{C}|\pi / 2+\varepsilon<| \arg \left(\lambda+\delta_{0}\right) \mid<\pi\right\} \cup\{0\} .
$$

Let $\delta>0$. Assume that the set $\Gamma$ consists of the parts $\Gamma_{1}$ and $\Gamma_{2}$ with

$$
\Gamma_{1}:=\left\{\lambda \in \mathbf{C} \mid \operatorname{Re} \lambda=-\delta, \operatorname{dist}\left(\lambda, \sigma\left(A^{0}\right)\right) \geq d_{0}\right\}
$$

with $d_{0}>0$ so large that $\Gamma_{1}$ consists of exactly two components. Let $\Gamma_{2} \subset \rho\left(A^{0}\right)$ be a compact pathwise connected set in the left half plane $\mathbf{C}^{-}$connecting the two components of $\Gamma_{1}$. Since the spectrum is upper half continuous with respect to generalized convergence [22, Theorem IV-3.1, p. 208], Theorem 4.12 yields $\Gamma_{2} \subset$ $\rho\left(A^{\tau}\right)$ for $0<\tau \leq \tau_{0}=\tau_{0}\left(\Gamma_{2}\right)$. It remains to show that for small $\tau>0$ the set $\Gamma_{1}$ belongs to $\rho\left(A^{\tau}\right)$. On $\Gamma_{1}$ the resolvent $\left(T_{0}-\lambda\right)^{-1}$ is uniformly bounded, and for the operator $R\left(\lambda, T^{\tau}\right)$ we calculate formally

$$
R\left(\lambda, T^{\tau}\right) \cdot\left[\operatorname{Id}-S_{\lambda}^{\tau} \cdot\left(T^{0}-\lambda\right)^{-1}\right]=\left(T^{0}-\lambda\right)^{-1}
$$

with $S_{\lambda}^{\tau}=T_{1}^{\tau}-T_{1}^{0}+e^{-\lambda \tau} T_{2}^{\tau}-T_{2}^{0}$ (cf. Definition 4.4). $S_{\lambda}^{\tau} \cdot\left(T^{0}-\lambda\right)^{-1}$ is a bounded operator whose norm vanishes uniformly for $\lambda \in \Gamma$ when $\tau \rightarrow 0$. So for small $\tau$ the operator in brackets is invertible, i.e. $R\left(\cdot, T^{\tau}\right)$ is defined as a holomorphic function on $\Gamma_{1}$, and because of Lemma 4.4 the proof is complete.

\section{REFERENCES}

1. D. G. Aronson, The asymptotic speed of propagation of a simple epidemic, Nonlinear Diffusion (W. E. Fitzgibbon and H. F. Walker, eds.), Research Notes in Math., no. 14, Pitman, London, 1977, pp. 1-23.

2. D. G. Aronson and H. F. Weinberger, Nonlinear diffusion in population genetics, combustion and nerve propagation, Partial Differential Equations and Related Topics (J. Goldstein, ed.), Lecture Notes in Math., vol. 446, Springer-Verlag, Berlin and New York, 1975, pp. 5-49.

3. __ Multidimensional nonlinear diffusion arising in population genetics, Adv. in Math. 30 (1978), 33-76.

4. C. Atkinson and G. E. H. Reuter, Deterministic epidemic waves, Math. Proc. Cambridge Philos. Soc. 80 (1976), 315-330.

5. M. Bramson, The convergence of solutions of the Kolmogorov equation to travelling waves, Mem. Amer. Math. Soc., no. 285, 1983.

6. E. A. Coddington and N. Levinson, Theory of ordinary differential equations, McGraw-Hill, New York, 1955.

7. H. Cohen, Non-linear diffusion problems, Studies in Applied Math., vol. 7, Math. Assoc. Amer., Washington, D. C., 1971.

8. O. Diekman, Thresholds and travelling waves for the geographical spread of infection, J. Math. Biol. 6 (1978), 109-130.

9. __ Run for your life. A note on the asymptotic speed of propagation of an epidemic, Math. Centre, Amsterdam, Report TW 176/78, 1978.

10. P. C. Fife, Mathematical aspects of reacting and diffusing systems, Lecture Notes in Biomath., vol. 28, Springer-Verlag, Berlin and New York, 1979. 
11. P. C. Fife and J. B. McLeod, The approach of solutions of nonlinear diffusion equations to travelling wave solutions, Bull. Amer. Math. Soc. 81 (1975), 1076-1078; Arch Rational Mech. Anal. 65 (1977), 335-361.

12. __ A phase plane discussion of convergence to travelling fronts for nonlinear diffusion, Arch. Rational Mech. Anal. 75 (1981), 281-314.

13. A. Friedman, Partial differential equations of parabolic type, Prentice-Hall, Englewood Cliffs, N. J., 1964.

14. I. C. Gohberg and M. G. Krein, Introduction to the theory of linear nonselfadjoint operators, Transl. Math. Monographs, vol. 18, Amer. Math. Soc., Providence, R. I., 1969.

15. K. P. Hadeler and F. Rothe, Travelling fronts in nonlinear diffusion equations, J. Math. Biol. 2 (1975), 251-263.

16. J. Hale, Theory of functional differential equations, 2nd ed., Appl. Math. Sci., vol. 3, SpringerVerlag, Berlin and New York, 1977.

17. D. Henry, Geometric theory of semilinear parabolic equations, Lecture Notes in Math., vol. 840, Springer-Verlag, Berlin and New York, 1981.

18. W. Jäger, H. Rost, and P. Tautu (eds), Biological growth and spread (Proc. Heidelberg 1979), Lecture Notes in Biomath., vol. 38, Springer-Verlag, Berlin and New York, 1980.

19. Y. Kametaka, On the nonlinear diffusion equations of Kolmogorov-Petrovsky-Piskunov type, Osaka J. Math. 13 (1976), 11-66.

20. Ja. I. Kanel', Stabilization of solutions of the Cauchy problem for equations encountered in combustion theory, Mat. Sb. (N.S.) 59(101) (1962), 245-288.

21. Mat. Sb. (N.S.) 65(107) (1964), 398-413.

22. T. Kato, Perturbation theory for linear operators, Spinger-Verlag, Berlin and New York, 1980.

23. K. Kobayashi, On the semilinear heat equation with time-lag, Hiroshima Math. J. 7 (1977), 459-472.

24. A. N. Kolmogoroff, I. G. Petrovski, and N. S. Piskunoff, Etude de l'équation de la diffusion avec croissance de la quantité de matière et son application à un problème biologique, Moscow Univ. Bull. Math., Série Internat., Sec. A, Math. et Méc. 1(6) (1937), 1-25.

25. H. P. McKean, Application of Brownian motion to the equation of KolmogorovPetrovskii-Piskunov, Comm. Pure Appl. Math. 28 (1975), 323-331.

26. R. Redheffer and W. Walter, Das Maximumprinzip in unbeschränkten Gebieten für parabolische Ungleichungen mit Funktionalen, Math. Ann. 226 (1977), 155-170.

27. F. Rothe, Convergence to travelling fronts in semilinear parabolic equations, Proc. Roy. Soc. Edinburgh Sect. A 80 (1978), 213-234.

28. D. Sattinger, On the stability of travelling waves, Adv. in Math. 22 (1976), 312-355.

29. W Weighted norms for the stability of travelling waves, J. Differential Equations 25 (1977), 179-201.

30. K. Schumacher, Travelling-front solutions for integro-differential equations. I, J. Reine Angew. Math. 316 (1980), 54-70.

31. __ Travelling-front solutions of integro-differential equations. II, Lecture Notes in Biomath., vol. 38, Springer-Verlag, Berlin and New York, 1980, pp. 296-309.

32. J. Szarski, Strong maximum principle for non-linear parabolic differential-functional inequalities in arbitrary domains, Ann. Polon. Math. 31 (1975), 197-203.

33. H. Thieme, Asymptotic estimates of the solutions of nonlinear integral equations and asymptotic speeds for the spread of populations, J. Reine Angew. Math. 306 (1979), 94-121.

34. K. Uchiyama, The behavior of solutions of some nonlinear diffusion equations for large time, J. Math. Kyoto Univ. 18 (1978), 453-508.

35. W. Walter, Differential and integral inequalities, Springer-Verlag, Berlin and New York, 1970.

36. H. F. Weinberger, Asymptotic behavior of a class of discrete time models in population genetics, Applied Nonlinear Analysis (V. Lakshmikantham, ed.), Academic Press, 1979, pp. 407-422.

37. E. M. Wright, The linear difference-differential equation with asymptotically constant coefficients, Amer. J. Math. 70 (1948), 221-238.

UNIVERSITÄT HEIDELBERG, SONDERFORSCHUNGSBEREICH 123, IM NEUENHEIMER FELD 294, D-6900 HEIDELBERG, FEDERAL REPUBLIC OF GERMANY 